Homology, Homotopy and Applications, vol.7(1), 2005, pp.87-108

\title{
MORE ABOUT THE (CO)HOMOLOGY OF GROUPS AND ASSOCIATIVE ALGEBRAS
}

\author{
HVEDRI INASSARIDZE
}

(communicated by Antonio Cegarra)

\begin{abstract}
It is proved that the homology and cohomology theories of groups and associative algebras are non-abelian derived functors of the cokernel and kernel groups of higher dimensions of their defining standard chain and cochain complexes respectively. The same results are also obtained for the relative (co)homology of groups, the mod q cohomology of groups and the cohomology of groups with operators. This allowed us to give an alternative approach to higher Hopf formulas for integral homology of groups. An axiomatic characterization of the relative cohomology of groups is given and higher relative $(n+1)$-th cohomology of groups is described in terms of $\mathrm{n}$-fold extensions.
\end{abstract}

\section{Introduction}

The present work deals with the investigation of the (co)homology of groups and associative algebras with respect to the first non-abelian variable. It is clear that this case is significantly more difficult to study than the case of the second abelian variable and fits into the theory of non-abelian homological algebra.

One of the fundamental theorems of non-abelian homological algebra says that homology and cohomology of groups and associative algebras can be expressed as non-abelian derived functors of the group Diff of differentials and the group Der of derivations respectively. That means by using projective resolutions with respect to the non-abelian variable and then by taking the functors Diff and Der with or into an appropriate module [1-4].

The groups Diff and Der are in fact the first cokernel and kernel groups respectively in the standard chain and cochain complexes determining the corresponding homology and cohomology of groups and associative algebras. Our aim is to develop the observation that the non-abelian derived functors of the groups of cokernel and kernel of higher dimensions of the above mentioned chain and cochain complexes

The work was partially supported by INTAS grant No 00-566, FNRS grant No 7GEPJO65513.01, NATO linkage grant PST.CLG.979167 and INTAS grant No 03-51-3251.

Received June 3, 2004, revised May 15, 2005; published on June 7, 2005.

2000 Mathematics Subject Classification: 18G10, 18G25,18G50, 18G55, 18 G60.

Key words and phrases: Cotriple derived functor, derivation, relative cohomology, crossed module. (C) 2005, Hvedri Inassaridze. Permission to copy for private use granted. 
respectively could be also used to describe the (co)homology of groups and associative algebras. In this context we shall consider the classical Eilenberg-MacLane (co)homology of groups (Theorems 1 and 3), the Hochschild (co)homology of associative algebras (Theorem 16), the relative cohomology of groups (Theorem 8), the mod q cohomology of groups defined in $[9,10]$ (Theorem 6) and the cohomology of groups with operators defined in [7] (Theorem 15). This approach to cotriple (co)homology theory is particularly needed to the investigation of the mod $q$ cohomology theory of groups (see [10]). Moreover these results allow us to give an alternative purely group theoretic description of the integral homology of groups (Theorem 4). By enlarging the category of coefficients we obtain an axiomatic characterization of the relative cohomology of groups (Theorem 11) and its description by $\mathrm{n}$-fold extensions in this wider class of coefficients (Theorem 14).

\section{Homology and cohomology of groups}

Let $\mathrm{G}$ be a group and $\mathrm{A}$ a $\mathrm{G}$-module. Let us consider the following standard cochain complex defining the cohomology of groups $H^{*}(G, A)$ :

$$
0 \rightarrow C^{0}(G, A) \rightarrow C^{1}(G, A) \rightarrow C^{2}(G, A) \rightarrow \cdots \rightarrow C^{n}(G, A) \rightarrow \cdots
$$

where $C^{0}(G, A)=A, C^{n}(G, A)$ being the group of maps $f: G^{n} \rightarrow A, n>0$, with coboundary operator $\delta^{n}: C^{n}(G, A) \rightarrow C^{n+1}(G, A)$ given by

$$
\begin{aligned}
& \delta^{n}(f)\left(x_{1}, \ldots, x_{n+1}\right)=x_{1} f\left(x_{2}, \ldots, x_{n+1}\right)+\sum(-1)^{i} f\left(x_{1}, \ldots, x_{i} x_{i+1}, \ldots, x_{n+1}\right)+ \\
& (-1)^{n+1} f\left(x_{1}, \ldots, x_{n}\right), \text { for } n>0 \text { and } \delta^{0}(a)=a-a x, x \in G .
\end{aligned}
$$

Denote $Z^{k}(G, A)=\operatorname{Ker} \delta^{k}$ and $B^{k}(G, A)=\operatorname{Im} \delta^{k}$ for $k \geqslant 0$. Clearly $Z^{0}(G, A)=$ $H^{0}(G, A)$ and $Z^{1}(G, A)=\operatorname{Der}(G, A)$. Then for any short exact sequence of $G$ modules

$$
0 \rightarrow A_{1} \rightarrow A \rightarrow A_{2} \rightarrow 0
$$

one has the following commutative diagram with exact rows and columns

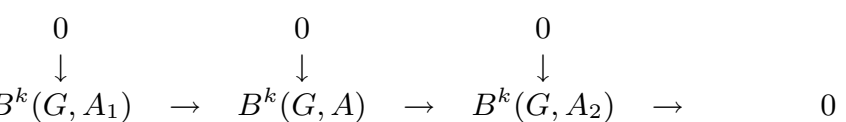

$$
\begin{aligned}
& 0 \\
& \begin{array}{c}
\downarrow \\
Z^{k}\left(G, A_{1}\right)
\end{array} \quad \rightarrow \quad Z^{k}(G, A) \quad \rightarrow \quad Z^{k}\left(G, A_{2}\right)
\end{aligned}
$$

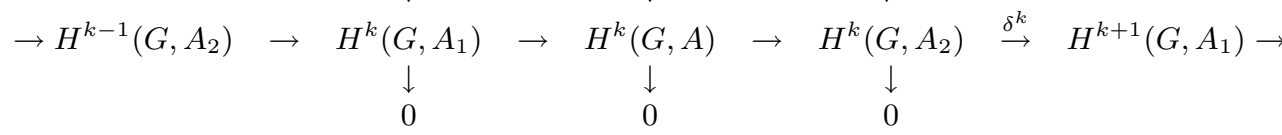

This diagram induces a long exact cohomology sequence

$$
0 \rightarrow Z^{k}\left(G, A_{1}\right) \rightarrow Z^{k}(G, A) \rightarrow Z^{k}\left(G, A_{2}\right) \rightarrow H^{k+1}\left(G, A_{1}\right) \rightarrow \cdots .
$$

Let $\mathcal{G}_{A}$ be the category of groups acting on an abelian group $A$ and $F: \mathcal{G}_{A} \rightarrow \mathcal{G}_{A}$ be the endofunctor which sends an object $G$ of $\mathcal{G}_{A}$ to the free group $F(G)$ on the underlying set of $G$, where the action of $F(G)$ on $A$ is defined by ${ }^{\left|g_{1}\right| \cdots\left|g_{i}\right|} a={ }^{g_{1} \cdots g_{i}} a$, 
$g_{i} \in G, a \in A$. Define natural morphisms $\tau_{G}: F(G) \rightarrow G$ and $\delta_{G}: F(G) \rightarrow F^{2}(G)=$ $F(F(G))$ of the category $\mathcal{G}_{A}$ by $\tau_{G}(|g|)=g$ and $\delta_{G}(|g|)=\|g\|, g \in G$. One gets a cotriple $\mathcal{F}=(F, \tau, \delta)$ on the category $\mathcal{G}_{A}$. Denote $F_{n}(G)=F^{n+1}(G)=F\left(F^{n}(G)\right)$ for $n \geqslant 0$. Therefore for any object $G$ of $\mathcal{G}_{A}$ one has the following free simplicial group $F_{*}(G)=\left\{F_{n}(G), \partial_{i}^{n}, s_{i}^{n}, n \geqslant 0\right\}$ acting on $A$, where $\partial_{i}^{n}=F_{i} \tau F_{n-i}$ and $s_{i}^{n}=F_{i} \delta F_{n-i}$.

Let $T: \mathcal{G}_{A} \rightarrow \mathfrak{A} b$ be a contravariant functor to the category $\mathfrak{A} b$ of abelian groups. Then the n-th homology group of the abelian cosimplicial group $T F_{*}(G)$ is called the n-th right derived functor $R_{\mathcal{F}}^{n} T$ of $T$ with respect to the cotriple $\mathcal{F}$. Let $\mathcal{P}$ be the projective class induced by $\mathcal{F}$, that means an object $X$ of $\mathcal{G}_{A}$ belongs to $\mathcal{P}$ if there is a morphism $\sigma: X \rightarrow F(X)$ such that $\tau \sigma=1_{X}$. Then the right derived functors $R_{\mathcal{P}}^{n} T$ of $T$ with respect to the projective class $\mathcal{P}$ are isomorphic to the cotriple derived functors $R_{\mathcal{F}}^{n} T$ (see $[23,15]$ ).

It is well known that the cotriple derived functors of the contravariant functor of derivations $\operatorname{Der}(-, A): \mathcal{G}_{A} \rightarrow \mathfrak{A} b$ are isomorphic, up to dimension shift, to the cohomology groups with coefficients in $A$ [1-4].

Theorem 1. There is a natural isomorphism

$$
R_{\mathcal{F}}^{n} Z^{k}(G, A) \approx H^{n+k}(G, A) \text { for all } n \geqslant 1 \text { and } k \geqslant 1 \text {. }
$$

Proof. The exact sequence (1) implies the exactness of the sequence

$$
0 \rightarrow Z^{k}\left(G, A_{1}\right) \rightarrow Z^{k}(G, A) \rightarrow Z^{k}\left(G, A_{2}\right) \rightarrow 0,
$$

if $G$ is a free group. One deduces that any short exact sequence $0 \rightarrow A_{1} \rightarrow A \rightarrow$ $A_{2} \rightarrow 0$ of $G$-modules yields a long exact sequence of derived functors

$$
\begin{aligned}
0 \rightarrow R_{\mathcal{F}}^{0} Z^{k}\left(G, A_{1}\right) \rightarrow & R_{\mathcal{F}}^{0} Z^{k}(G, A) \rightarrow R_{\mathcal{F}}^{0} Z^{k}\left(G, A_{2}\right) \rightarrow R_{\mathcal{F}}^{1} Z^{k}\left(G, A_{1}\right) \rightarrow \\
& R_{\mathcal{F}}^{1} Z^{k}(G, A) \rightarrow R_{\mathcal{F}}^{1} Z^{k}\left(G, A_{2}\right) \rightarrow R_{\mathcal{F}}^{2} Z^{k}\left(G, A_{1}\right) \rightarrow \cdots
\end{aligned}
$$

Now it will be shown that one has a natural isomorphism $R_{\mathcal{F}}^{0} Z^{k}(-, A) \approx Z^{k}(-, A)$, $k>0$.

Let $F_{*}(G) \rightarrow G$ be the free cotriple resolution of $G$. Since it is left (right) contractible as augmented simplicial set (for the categorical definition of left contractibility (see $[22,15]$ ), it follows that the augmented abelian simplicial group $\left(C^{i}\left(F_{*}(G), A\right) \rightarrow C^{i}(G, A)\right)$ is left contractible in the category of abelian groups for all $i \geqslant 0$ and therefore the groups $H_{n}\left(C^{i}\left(F_{*}(G), A\right)\right)$ are trivial for $n>0$ and $H_{0}\left(C^{i}\left(F_{*}(G), A\right)\right) \approx C^{i}(G, A)$ for all $i \geqslant 0$.

The diagram

$$
\begin{aligned}
& 0 \rightarrow \quad Z^{k}(F(G), A) \quad \rightarrow \quad C^{k}(F(G), A) \quad \rightarrow \quad \operatorname{Im} \delta_{F(G)}^{k} \quad \rightarrow \quad C^{k+1}(F(G), A)
\end{aligned}
$$

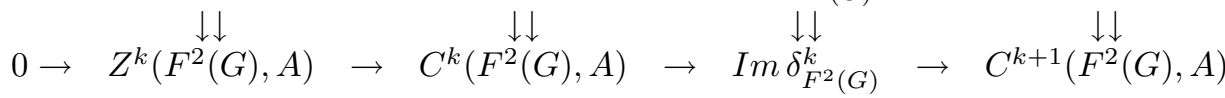

implies an exact sequence

$$
R_{\mathcal{F}}^{0} Z^{k}(G, A) \rightarrow R_{\mathcal{F}}^{0} C^{k}(G, A) \rightarrow R_{\mathcal{F}}^{0} \operatorname{Im} \delta_{G}^{k} \rightarrow R_{\mathcal{F}}^{1} Z^{k}(G, A),
$$

where $R_{\mathcal{F}}^{0} C^{k}(G, A) \approx C^{k}(G, A)$ and one has an injection $R_{\mathcal{F}}^{0} \operatorname{Im} \delta_{G}^{k} \rightarrow C^{k+1}(G, A)$. It follows that $R_{\mathcal{F}}^{0} Z^{k}(G, A) \approx K \operatorname{er} \delta_{G}^{k}$. 
Therefore in the exact sequence (3) the zero-derived functors could be replaced by the functors $Z^{k}(G,-)$.

Further it will be shown that $R_{\mathcal{F}}^{n} Z^{k}(G, A)=0$ for $n, k>0$, if $A$ is a coinduced $G$-module. In effect, to the free cotriple resolution $F_{*}(G) \rightarrow G$ of the group $G$ apply the standard cochain complex $\left\{C^{*}(G, A), \delta^{n}\right\}$ to get the following cochain complex of abelian cosimplicial groups:

$$
\begin{aligned}
0 \rightarrow C^{0}\left(F_{*}(G), A\right) \stackrel{\widetilde{\delta_{*}^{0}}}{\rightarrow} C^{1}\left(F_{*}(G), A\right) \stackrel{\widetilde{\delta_{*}^{1}}}{\rightarrow} C^{2}\left(F_{*}(G), A\right) \stackrel{\widetilde{\delta_{*}^{2}}}{\rightarrow} \\
C^{3}\left(F_{*}(G), A\right) \stackrel{\widetilde{\delta_{*}^{3}}}{\rightarrow} \cdots \stackrel{\widetilde{\delta_{*}^{k-1}}}{\rightarrow} C^{k}\left(F_{*}(G), A\right) \stackrel{\widetilde{\delta_{*}^{k}}}{\rightarrow} \cdots .
\end{aligned}
$$

Since each $F_{i}(G), i \geqslant 0$, is a free group, the sequence (6) is exact except at the terms $C^{0}$ and $C^{1}$ for any $G$-module $A$.

The short exact sequence of abelian cosimplicial groups

$$
0 \rightarrow \operatorname{Der}\left(F_{*}(G), A\right) \rightarrow C^{1}\left(F_{*}(G), A\right) \rightarrow \operatorname{Im}\left(C^{1} F_{*}\left(\delta^{1}\right)\right) \rightarrow 0
$$

yields the isomorphism $H_{n}\left(\operatorname{Im} \widetilde{\delta_{*}^{1}}\right) \approx H_{n+1}\left(\operatorname{Der}\left(F_{*}(G), A\right)\right.$ for $n>0$ and therefore, by the exactness of $(6)$, the isomorphism $H_{n}\left(\operatorname{Ker} \widetilde{\delta}_{*}^{2}\right) \approx H_{n+1}\left(\operatorname{Der}\left(F_{*}(G), A\right)\right.$ for $n>0$.

Consider now the short exact sequence of abelian cosimplicial groups

$$
0 \rightarrow \operatorname{Ker}\left(\tilde{\delta}_{*}^{2}\right) \rightarrow C^{2}\left(F_{*}(G), A\right) \rightarrow \operatorname{Im}\left(\tilde{\delta}_{*}^{2}\right) \rightarrow 0
$$

that gets the isomorphism $H_{n}\left(\operatorname{Im} \widetilde{\delta_{*}^{2}}\right) \approx H_{n+1}\left(\operatorname{Ker} \widetilde{\delta_{*}^{2}}\right)$ for $n>0$, implying the isomorphism $H_{n}\left(\operatorname{Ker}\left(\widetilde{\delta_{*}^{3}}\right) \approx H_{n+2}\left(\operatorname{Der}\left(F_{*}(G), A\right)\right)\right.$ for $n>0$ and any $G$-module $A$. Continuing in this way, by using the exact sequence (6) and the triviality of the homology groups $H_{n}\left(C^{k}\left(F_{*}(G), A\right)\right), n>0$, one arrives to the isomorphism $H_{n}\left(\operatorname{Ker}\left(C^{k}\left(F_{*}(G), A\right)\right) \approx H_{n+k-1}\left(\operatorname{Der}\left(F_{*}(G), A\right)\right)\right.$ for $k>1, n>0$, and any $G$ module $A$. Suppose $A$ is a coinduced $G$-module. In this case it is well known that the groups $H_{n}\left(\operatorname{Der}\left(F_{*}(G), A\right)\right.$ are trivial for $n>0$. Thus we deduce the required triviality of the derived functors $R_{\mathcal{F}}^{n} Z^{k}(G, A)$ for $k, n>0$, when $A$ is a coinduced $G$-module.

The rest of the proof is standard by using the exact sequences (2) and (4) and the fact that both functors $H^{n}(-, A)$ and $R_{\mathcal{F}}^{n} Z^{k}(-, A)$ vanish for $k, n>0$, if $A$ is a coinduced $G$-module.

Let

$$
C_{*}(G)=\cdots \rightarrow C_{3}(G) \rightarrow C_{2}(G) \rightarrow C_{1}(G) \rightarrow C_{0}(G) \rightarrow \mathbb{Z} \rightarrow 0
$$

be the standard bar resolution of $\mathbb{Z}$ and $C_{*}(G, A)=C_{*}(G) \otimes_{G} A$ the defining standard complex for the homology of groups with coefficients in the $G$-module $A$. Denote $Y_{k}(G)=$ Coker $\partial_{k+1}$ and $Y_{k}(G, A)=Y_{k}(G) \otimes_{G} A$ for $k \geqslant 1$, where $\partial_{k}: C_{k+1}(G) \rightarrow C_{k}(G), k \geqslant 0$, and $\varepsilon: C_{0}(G) \rightarrow \mathbb{Z}$ are homomorphisms of the bar resolution (9). Clearly $Y_{k}(G, A) \approx \operatorname{Coker}\left(\partial_{k+1} \otimes_{G} A\right), Y_{0}(G, A)=H_{0}(G, A)$ and $Y_{1}(G, A)=I(G) \otimes_{G} A$. It is well known [2] that the homology group functors $H_{n}(-, A), n>1$, are isomorphic, up to dimension shift, to the non-abelian left 
derived functors of the functor $I(-) \otimes_{G} A$ with respect to the free cotriple $\mathcal{F}$ for $n>1$.

Proposition 2. There is an isomorphism

$$
\underset{G}{\operatorname{Hom}}\left(Y_{k}(G), A\right) \approx Z^{k}(G, A) \text { for } k \geqslant 1 .
$$

Proof. For $k=1$ this isomorphism is well known. Take the short exact sequence

$$
0 \rightarrow I m \partial_{k+1} \rightarrow C_{k}(G) \rightarrow Y_{k}(G) \rightarrow 0
$$

induced by the sequence (9) for $k \geqslant 1$. One gets an exact sequence

$$
\begin{gathered}
0 \rightarrow \underset{G}{\operatorname{Hom}}\left(Y_{k}(G), A\right) \rightarrow \underset{G}{\operatorname{Hom}}\left(C_{k}(G), A\right) \rightarrow \\
\underset{G}{\operatorname{Hom}}\left(\operatorname{Im} \partial_{k+1}, A\right) \rightarrow \underset{G}{\operatorname{Ext}}\left(Y_{k}(G), A\right) \rightarrow 0 .
\end{gathered}
$$

It follows that

$$
\operatorname{Ker}\left(\underset{G}{\operatorname{Hom}}\left(C_{k}(G), A\right) \rightarrow \underset{G}{\operatorname{Hom}}\left(\operatorname{Im} \partial_{k+1}, A\right)\right)=Z^{k}(G, A),
$$

implying the required isomorphism for $k \geqslant 1$.

Theorem 3. There is a natural isomorphism

$$
L_{n}^{\mathcal{F}} Y_{k}(G, A) \approx H_{n+k}(G, A) \text { for all } n \geqslant 1 \text { and } k \geqslant 1 \text {. }
$$

Proof. Completely similar to the proof of Theorem 1 and will be omitted.

Now an application of Theorem 3 will be given in connection with the purely group theoretic interpretation of the integral homology of groups. Such description in low dimensions is well known, since one has $H_{1}(G) \approx G /[G, G]$ and $H_{2}(G) \approx$ $(R \cap[F, F]) /[F, R]$ (Hopf formula), where $R$ is the kernel of a free presentation $F \rightarrow G$ of the group $G$.

Different approaches to this problem for higher integral homology of groups have been given in $[6,11,12,20,21]$ by constructing higher Hopf type formulas. By using Theorem 3 we provide an alternative group theoretic interpretation of the integral homology $H_{*}(G)$ of a group $G$.

Let us consider the natural surjective homomorphisms $\alpha_{n+1}: C_{n+1}(G) \otimes_{G}$ $\mathbb{Z} \rightarrow Y_{n+1}(G, \mathbb{Z})$ induced by $\partial_{n+1}, n \geqslant 0$. Clearly $C_{n+1}(G) \otimes_{G} \mathbb{Z}$ is isomorphic to $\Sigma_{\alpha \in G^{n+1}} \mathbb{Z}_{\alpha}, \mathbb{Z}_{\alpha} \approx \mathbb{Z}$, and the abelianization of $F\left(G^{n+1}\right)$ is isomorphic to $\Sigma_{\alpha \in G^{n+1}} \mathbb{Z}_{\alpha}$. Thus one gets functorial surjective homomorphisms

$$
F\left(G^{n+1}\right) \stackrel{\beta_{n+1}}{\rightarrow} C_{n+1}(G) \otimes_{G} \mathbb{Z} \stackrel{\alpha_{n+1}}{\rightarrow} Y_{n+1}(G, \mathbb{Z}), \quad n \geqslant 0,
$$

where $\beta_{n+1}$ is the composite of the homomorphisms $F\left(G^{n+1}\right) \rightarrow F\left(G^{n+1}\right)^{a b}$, $F\left(G^{n+1}\right)^{a b} \approx \Sigma \mathbb{Z}_{\alpha}$ and $\Sigma \mathbb{Z}_{\alpha} \approx C_{n+1}(G) \otimes_{G} \mathbb{Z}$.

Finally we obtain a functorial surjective homomorphism

$$
\gamma_{n+1}(G)=\alpha_{n+1} \beta_{n+1}: F\left(G^{n+1}\right) \rightarrow Y_{n+1}(G, \mathbb{Z}), \quad n \geqslant 0,
$$

and denote $U_{n+1}(G)=\operatorname{Ker} \gamma_{n+1}(G)$ for all $n \geqslant 0$. Therefore $U_{k}(G)=\left[F\left(G^{k}\right), F\left(G^{k}\right)\right]$ for $k \geqslant 1$. The functors $U_{k}, k \geqslant 1$, will be used to provide higher Hopf type formulas. 
Let

$$
F_{*, G} \stackrel{\tau}{\longrightarrow} G=\quad \cdots \underset{\vdots}{\longrightarrow} F_{2} \underset{\partial_{2}^{2}}{\stackrel{\partial_{0}^{2}}{\longrightarrow}} F_{1} \underset{\partial_{1}^{1}}{\stackrel{\partial_{0}^{1}}{\rightleftarrows}} F_{0} \stackrel{\tau}{\longrightarrow} G
$$

be a $\mathcal{P}$-projective resolution of $G$, that means this augmented pseudosimplicial group is simplicially exact and the groups $F_{i}, i \geqslant 0$, are free groups. The projective class $\mathcal{P}$ is induced by the free cotriple in the category $\mathcal{G}$ of groups. Thus $\mathcal{P}$ is consisting of free groups and a morphism of $\mathcal{G}$ is a $\mathcal{P}$-epimorphism if and only if it is an epimorphism. It is known that the free cotriple resolution of the group $G$ is a $\mathcal{P}$ projective resolution of $G$ and any free presentation $\tau: F \rightarrow G$ of $G$ induces a well defined $\mathcal{P}$-projective resolution $P_{*} \stackrel{\tau}{\rightarrow} G$ of $G$ by using simplicial kernels [12] and constructed as follows:

$$
\ldots \quad \underset{l_{3}^{3}}{\stackrel{l_{0}^{3}}{\leftrightarrows}} F\left(L_{2} G\right) \stackrel{\tau_{L_{2} G}}{\longrightarrow} L_{2} G \underset{l_{2}^{2}}{\stackrel{l_{0}^{2}}{\longrightarrow}} F\left(L_{1} G\right) \stackrel{\tau_{L_{1} G}}{\longrightarrow} L_{1} G \stackrel{\stackrel{l_{0}^{1}}{\longrightarrow}}{\longrightarrow} F \stackrel{\tau}{\longrightarrow} G,
$$

where $\left(L_{1} G, l_{0}^{1}, l_{1}^{1}\right)$ is the simplicial kernel of the morphism $\tau,\left(L_{2} G, l_{0}^{2}, l_{1}^{2}, l_{2}^{2}\right)$ is the kernel of the pair of morphisms $\left(l_{0}^{1} \tau_{L_{1} G}, l_{1}^{1} \tau_{L_{1} G}\right)$ and if $\left(L_{n} G, l_{0}^{n}, \ldots, l_{n}^{n}\right)$ has been constructed, then $\left(L_{n+1} G, l_{0}^{n+1}, \ldots, l_{n+1}^{n+1}\right)$ is the simplicial kernel of the sequence of morphisms $\left(l_{0}^{n} \tau_{L_{n} G}, \ldots, l_{n}^{n} \tau_{L_{n} G}\right)$. Denote $P_{0}=F, P_{n}=F\left(L_{n} G\right)$ and $\partial_{i}^{n}=l_{i}^{n} \tau_{L_{n} G}$ for $n>0$.

Theorem 4. For any $\mathcal{P}$-projective resolution $\left(F_{*, G} \stackrel{\tau}{\rightarrow} G, \partial_{i}^{n}, s_{i}^{n}\right)$ of the group $G$ there are isomorphisms

$$
H_{k+1}(G) \approx\left(R_{k} \cap U_{k}\left(F_{0}\right)\right) / U_{k}\left(\partial_{1}^{1}\right)\left(\operatorname{Ker} U_{k}\left(\partial_{0}^{1}\right)\right), \quad k \geqslant 1,
$$

where $R_{k}=\operatorname{Ker} F\left(\tau_{k}\right)$ and $\tau_{k}: F_{0}^{k} \rightarrow G^{k}$ is induced by $\tau, k \geqslant 1$.

Proof. By applying the functors $U_{k}, k \geqslant 1$, one gets a short exact sequence of augmented pseudosimplicial (simplicial) groups

$$
\begin{gathered}
1 \rightarrow\left(U_{k}\left(F_{*, G}\right) \stackrel{U_{k}(\tau)}{\rightarrow} U_{k}(G)\right) \rightarrow\left(F\left(F_{*, G}^{k}\right) \stackrel{F\left(\tau^{k}\right)}{\rightarrow} F\left(G^{k}\right)\right) \rightarrow \\
\left.\left(Y_{k}\left(F_{*, G}, \mathbb{Z}\right)\right) \stackrel{Y_{k}(\tau, \mathbb{Z})}{\longrightarrow} Y_{k}(G, \mathbb{Z})\right) \rightarrow 1,
\end{gathered}
$$

for $k \geqslant 1$ which induces a long exact homotopy sequence. It is easily checked that the augmented pseudosimplicial group $F_{*, G} \stackrel{\tau}{\rightarrow} G$ is left contractible as an augmented pseudosimplicial set. Thus the augmented pseudosimplicial group $F_{*, G}^{k} \stackrel{\tau^{k}}{\rightarrow} G^{k}$ is also left contractible in the category of sets implying the left contractibility of $F\left(F_{*, G}^{k}\right) \stackrel{F\left(\tau^{k}\right)}{\rightarrow} F\left(G^{k}\right)$ in the category of groups. It follows that $\pi_{n}\left(F\left(F_{*, G}^{k}\right)\right)=0$ for $n>0$ and $\pi_{0}\left(F\left(F_{*, G}^{k}\right)\right)$ is naturally isomorphic to $F\left(G^{k}\right)$. Therefore we have the following exact sequence

$$
0 \rightarrow \pi_{1} Y_{k}\left(F_{*, G}, \mathbb{Z}\right) \rightarrow \pi_{0} U_{k}\left(F_{*, G}\right) \rightarrow \pi_{0}\left(F\left(F_{*, G}^{k}\right)\right)
$$

for $k \geqslant 1$. By Theorem 3 one has an isomorphism $\pi_{1} Y_{k}\left(F_{*, G}, \mathbb{Z}\right) \approx H_{k+1}(G), k \geqslant 1$. 
Finally we obtain an exact sequence

$$
0 \rightarrow H_{k+1}(G) \rightarrow \pi_{o} U_{k}\left(F_{*, G}\right) \rightarrow F\left(G^{k}\right)
$$

which gives the desired result.

Remark 5. The group $\left(R_{k} \cap U_{k}\left(F_{0}\right)\right) / U_{k}\left(\partial_{1}^{1}\right)\left(\operatorname{Ker} U_{k}\left(\partial_{0}^{1}\right)\right), k \geqslant 1$, does not depend of the projective resolution of the group $G$.

For $k=1,2$ instead of the functors $F(G)$ and $F\left(G^{2}\right)$ we can take the identity functor $1_{G}$ and the kernel $R_{G}$ of the canonical map $\gamma: F(G) \rightarrow G$ respectively. Denote $U_{1}^{\prime}(G)=[G, G]$ and $U_{2}^{\prime}(G)=\operatorname{Ker} \nu$, where $\nu:[F(G), F(G)] \rightarrow[G, G]$ is induced by $\gamma$. Then there are isomorphisms

$$
H_{k+1}(G) \approx\left(R_{k} \cap U_{k}^{\prime}\left(F_{0}\right)\right) / U_{k}^{\prime}\left(\partial_{1}^{1}\right)\left(\operatorname{Ker} U_{k}^{\prime}\left(\partial_{0}^{1}\right)\right), \quad k=1,2,
$$

where $R_{1}$ is the kernel of $\tau$ and $R_{2}$ is the kernel of $F(\tau)$. It can be proved that $\left(R_{1} \cap U_{1}^{\prime}\left(F_{0}\right)\right) / U_{1}^{\prime}\left(\partial_{1}^{1}\right)\left(\operatorname{Ker} U_{1}^{\prime}\left(\partial_{0}^{1}\right)\right)$ is the Hopf formula by showing the equality

$$
U_{1}^{\prime}\left(\partial_{1}^{1}\right)\left(\operatorname{Ker} U_{1}^{\prime}\left(\partial_{0}^{1}\right)\right)=\left[F_{0}, R_{1}\right]
$$

(see [16]). The sequence $0 \rightarrow U_{1}^{\prime}(G) \rightarrow G \rightarrow Y_{1}(G, \mathbb{Z}) \rightarrow 0$ is exact for any group $G$ and the sequence $0 \rightarrow U_{2}^{\prime}(F) \rightarrow R_{G} \rightarrow Y_{2}(F, \mathbb{Z}) \rightarrow 0$ is exact for any free group $F$, where the homomorphism $R_{G} \rightarrow Y_{2}(F, \mathbb{Z})$ is defined as follows. For any group $G$ one has a surjective homomorphism $\beta_{1}^{\prime}: R_{G} \rightarrow \operatorname{Ker} \alpha_{1}$ induced by $\beta_{1}$ and a surjective homomorphism $\vartheta: Y_{2}(G, \mathbb{Z}) \rightarrow \operatorname{Ker} \alpha_{1}$ which is an isomorphism if $G$ is a free group. Then the required homomorphism $R_{G} \rightarrow Y_{2}(F, \mathbb{Z})$ is equal to the composite $\vartheta^{-1} \beta_{1}^{\prime}$. Using these short exact sequences the proof of the afore given isomorphisms for $k=1,2$ is completely similar to the proof of Theorem 4 .

We shall not consider in this setting the case of Lie algebras, but it should be noted that for the (co)homology of Lie algebras results similar to Theorems 2 and 3 with respect to the standard Chevalley- Eilenberg complex can be proved and by the same way higher Hopf type formulas can be obtained for the homology $H_{*}(L, k)$ of a Lie algebra $L$ over $k$.

\section{Mod $q$ cohomology of groups}

This case has been already investigated in [10], Section 2. However, in this context it is an important example showing the necessity to use the non-abelian derived functors of higher dimensional kernels of the defining cochain complex to express the corresponding cohomology groups as cotriple cohomology. That is the reason why a short expository will be given.

The $\bmod q$ cohomology $H^{n}\left(G, A, \mathbb{Z}_{q}\right), n \geqslant 0$, of a group $G$ with coefficients in a $G$-module $A$ is defined by $H^{n}\left(G, A, \mathbb{Z}_{q}\right)=\operatorname{Ext}_{G}^{n}\left(\mathbb{Z}_{q}, A\right)$ with $Z_{q}=\mathbb{Z} / q$ as a trivial $G$-module $[9,10]$.

The standard cochain complex for the $\bmod q$ cohomology of groups is given by the cochain complex $\left(\operatorname{Hom}_{G}\left(q-C_{*}, A\right), \tilde{\delta^{*}}\right)$, where $C_{*} \rightarrow \mathbb{Z}$ is the standard bar 
resolution of the trivial $G$-module $\mathbb{Z}$ and

$$
\begin{aligned}
q-C_{*} \rightarrow \mathbb{Z}_{q} \equiv \cdots \longrightarrow C_{n+1} \oplus C_{n} \stackrel{\widetilde{\partial_{n+1}}}{\longrightarrow} C_{n} \oplus C_{n-1} \stackrel{\widetilde{\partial_{n}}}{\longrightarrow} C_{n-1} \oplus C_{n-2} \stackrel{\widetilde{\partial_{n-1}}}{\longrightarrow} \cdots \\
\stackrel{\widetilde{\partial_{2}}}{\longrightarrow} C_{1} \oplus C_{0} \stackrel{\widetilde{\partial_{1}}}{\longrightarrow} C_{0} \stackrel{\widetilde{\epsilon}}{\longrightarrow} \mathbb{Z}_{q} \longrightarrow 0,
\end{aligned}
$$

is a free $\mathbb{Z}[G]$-resolution of the trivial $G$-module $\mathbb{Z}_{q}$ with

$$
\widetilde{\partial_{n}}\left(x_{n}, x_{n-1}\right)=\left(\partial_{n}\left(x_{n}\right)+q x_{n-1},-\partial_{n-1}\left(x_{n-1}\right)\right)
$$

for $\left.n \geqslant 2, \widetilde{\partial}_{1}\left(x_{1}\right), x_{0}\right)=\partial_{1}\left(x_{1}\right)+q x_{0}$ and $\widetilde{\epsilon}\left(x_{0}\right)=\left[\epsilon\left(x_{0}\right)\right]$.

For $\bmod q$ cohomology of groups one has the following universal coefficient formula:

$$
0 \rightarrow H^{n-1}(G, A) \otimes \mathbb{Z}_{q} \rightarrow H^{n}\left(G, A, \mathbb{Z}_{q}\right) \rightarrow \operatorname{Tor}\left(H^{n}(G, A), \mathbb{Z}_{q}\right) \rightarrow 0
$$

for all $n \geqslant 0$, where $H^{-1}(G, A)=0$.

The cotriple derived functor $R_{\mathcal{F}}^{n} q-Z^{1}(-, A)$ of the functor $q-Z^{1}(-, A)$ of $q$ derivations is not isomorphic to the $\bmod q$ group cohomology functor $H^{n+1}\left(-, A, \mathbb{Z}_{q}\right)$ for some $n \geqslant 1$. In effect, if $F$ is a free group acting on $A$, then $R_{\mathcal{F}}^{1} q-Z^{1}(F, A)=0$, while the group $H^{2}\left(F, A, \mathbb{Z}_{q}\right)$ is isomorphic to $H^{1}(F, A) \otimes \mathbb{Z}_{q}$. But the following assertion holds [10].

Theorem 6. Let $G$ be a group, $A$ be a $G$-module and $q$ a positive integer. Then there are natural isomorphisms

$$
\begin{aligned}
& R_{\mathcal{F}}^{0}\left(q-Z^{k}\right)(G, A) \approx q-Z^{k}(G, A), \\
& R_{\mathcal{F}}^{n}\left(q-Z^{k}\right)(G, A) \approx H^{n+k}\left(G, A, \mathbb{Z}_{q}\right) \text { for all } k>1, n>0 .
\end{aligned}
$$

Proof. A sketch will be given. Let us denote the groups $\operatorname{Ker} \widetilde{\delta^{k}}$ and $\operatorname{Im} \widetilde{\delta^{k}}$ by $q-$ $Z^{k}(G, A)$ and $q-B^{k+1}(G, A)$ respectively for $k \geqslant 0$. Clearly, $q-Z^{0}(G, A)=q-$ $H^{0}(G, A)$ and $\operatorname{Ker} \tilde{\delta}^{1}$ is the group of $q$-derivations from $G$ to $A$. It is shown that any short exact sequence of $G$-modules induces a long exact $\bmod q$ cohomology sequence and a long exact sequence of cotriple derived functors $R_{\mathcal{F}}^{n}\left(q-Z^{k}\right)(-, A)$ for $k>1$. By using the $q$-divisibility of injective $G$-modules it is proved that $R_{\mathcal{F}}^{n}\left(q-Z^{k}\right)(G, A)=0$ for $k \geqslant 1$ and $n>0$, if $A$ is an injective $G$-module. The rest of the proof is standard.

By Theorem 6 the mod $q$ cohomology groups $H^{3}\left(G, A, \mathbb{Z}_{q}\right), H^{4}\left(G, A, \mathbb{Z}_{q}\right), \ldots$, $H^{2+n}\left(G, A, \mathbb{Z}_{q}\right), \ldots$ can be expressed in terms of the cotriple derived functors $R_{\mathcal{F}}^{n}\left(q-Z^{2}\right)(-, A)$ of the contravariant functor $q-Z^{2}(-, A)$.

\section{Relative (co)homology of groups}

Let $f: G \rightarrow G^{\prime}$ be a group homomorphism. It will be said that an abelian group $A$ is an $f$-module, if the groups $G$ and $G^{\prime}$ act on $A$ and $g a=f(g) a$ for any $g \in G$ and $a \in A$. If $A$ is an $f$-module with $f$ surjective, by definition the homology groups of the standard cochain complex $C^{*}(f, A)=C^{*}(G, A) / C^{*}\left(G^{\prime}, A\right)$ are called the relative cohomology groups $H^{n}(f, A), n \geqslant 0$, of the surjective homomorphism $f$ with 
coefficients in the $f$-module $A$. Similarly, the homology groups of the standard chain complex $C_{*}(f, A)=\operatorname{Ker}\left(f_{*}: C_{*}(G, A) \rightarrow C_{*}\left(G^{\prime}, A\right)\right)$ are called the relative homology groups $H_{n}(f, G), n \geqslant 0$, of the surjective homomorphism $f$ with coefficients in the $f$-module $A$.

There is another cocycle description of this defining cochain complex for the relative cohomology of groups. Let $\left(L, l_{0}, l_{1}\right)$ be the simplicial kernel of $f$. Then it is easily checked that the subcomplex of $C^{*}(L, A)$, consisting of all cochains $\psi$ such that there exists a cochain $\eta \in C^{*}(G, A)$ satisfying the equality $l_{0} \eta-l_{1} \eta=\psi$, is isomorphic to $C^{*}(f, A)$.

Denote by $Z^{k}(f, A)$ the kernel of the differential $\tilde{\delta^{k}}$ of the cochain complex $C^{*}(f, A)$ for $k \geqslant 0$.

Definition 7. The group $\operatorname{Der}(f, A)$ consisting of all crossed homomorphisms $f \in$ $Z^{1}(L, A)$ such that $f(x, x)=0, x \in G$, is called the group of derivations of the surjective homomorphism $f: G \rightarrow G^{\prime}$.

It is easily checked that $\operatorname{Der}(f, A)$ is naturally isomorphic to $Z^{1}(f, A)$.

Let $\mathcal{M G}_{A}$ be the category whose objects are surjective homomorphisms (epimorphisms) of groups acting on a fixed abelian group $A$ and morphisms are couples $\left(\alpha, \alpha^{\prime}\right): f \rightarrow f^{\prime}$ of group homomorphisms such that $f^{\prime} \alpha=\alpha^{\prime} f$. The free cotriple $\mathcal{F}=(F, \delta, \tau)$ defined in the category $\mathcal{G}_{A}$ induces in a natural way a cotriple $\mathcal{M F}$ given by $M F(f)=F(f)$ and $M F\left(\alpha, \alpha^{\prime}\right)=\left(F(\alpha), F\left(\alpha^{\prime}\right)\right)$ for any object $f$ and the natural transformations of this cotriple are induced by the natural transformations of the free cotriple $\mathcal{F}$.

Theorem 8. There is a natural isomorphism

$$
R_{\mathcal{M F}}^{n} Z^{k}(f, A) \approx H^{n+k}(f, A)
$$

for all $n \geqslant 1$ and $k \geqslant 1$.

Proof. The long exact relative cohomology sequence yields a short exact sequence

$$
0 \rightarrow Z^{k}\left(G^{\prime}, A\right) \rightarrow Z^{k}(G, A) \rightarrow Z^{k}(f, A) \rightarrow 0
$$

for any $k \geqslant 1$ and $f: G \rightarrow G^{\prime}$ with $G^{\prime}$ a free group, since in this case $H^{n}\left(G^{\prime}, A\right)=0$, $n \geqslant 2$. Therefore, for any object $f$ of the category $\mathcal{M F}_{A}$ one has a long exact sequence of non-abelian right derived functors

$$
\begin{aligned}
0 \rightarrow R_{\mathcal{F}}^{0} Z^{k}\left(G^{\prime}, A\right) & \rightarrow R_{\mathcal{F}}^{0} Z^{k}(G, A) \rightarrow R_{\mathcal{M F}}^{0} Z^{k}(f, A) \rightarrow \\
R_{\mathcal{F}}^{1} Z^{k}\left(G^{\prime}, A\right) \rightarrow \cdots \rightarrow R_{\mathcal{M F}}^{n-1} Z^{k}(f, A) & \rightarrow R_{\mathcal{F}}^{n} Z^{k}\left(G^{\prime}, A\right) \rightarrow \\
R_{\mathcal{F}}^{n} Z^{k}(G, A) & \rightarrow R_{\mathcal{M} \mathcal{F}}^{n} Z^{k}(f, A) \rightarrow R_{\mathcal{F}}^{n+1} Z^{k}\left(G^{\prime}, A\right) \rightarrow \cdots
\end{aligned}
$$

where the zero-derived functors could be replaced by the corresponding functors $Z^{k}(-, A)$.

Let $C_{k}^{*}(G, A)$ be the cochain complex defined by $C_{k}^{n}(G, A)=C^{n+k}(G, A), n \geqslant$ $0, k \geqslant 1$. It is clear that $H_{0}\left(C_{k}^{*}(G, A)\right)=Z^{k}(G, A)$ and if $G$ is a free group, the homology groups $H_{n}\left(C_{k}^{*}(G, A)\right)$ are trivial for $n \geqslant 1$. This definition could be extended to any object $f$ of the category $\mathcal{M F}_{A}$ by $C_{k}^{*}(f, A)=C_{k}^{*}(G, A) / C_{k}^{*}\left(G^{\prime}, A\right)$ 
with $f: G \rightarrow G^{\prime}$. Take now the free cotriple resolution $F_{*}(f)$ of $f$ with $F_{i}(f)=$ $F_{i}(G) \rightarrow F_{i}\left(G^{\prime}\right), i \geqslant 0$. Then the following equalities hold:

$H_{n}\left(C_{k}^{*}\left(F_{i}(f), A\right)=0, n>0\right.$, and $H_{0}\left(C^{*}\left(F_{i}(f), A\right)=Z^{k}\left(F_{i}(f), A\right)\right.$ for all $i \geqslant 0$, since the groups $F_{i}(G)$ and $F_{i}\left(G^{\prime}\right)$ are free groups; $H_{n}\left(C_{k}^{j}\left(F_{*}(f), A\right)=0, n>0\right.$, and $H_{0}\left(C_{k}^{j}\left(F_{*}(f), A\right)=C_{k}^{j}(f, A)\right.$ for all $j \geqslant 0$.

Therefore, both homology groups $H_{n}\left(Z^{k}\left(F_{*}(f), A\right)\right)$ and $H_{n}\left(C_{k}^{*}(f, A)\right)$ are isomorphic for all $n, k>0$ to the $n$-th homology group of the total cochain complex $\operatorname{Tot}\left(C_{k}^{*}\left(F_{*}(f), A\right)\right)$ of the bicomplex $C_{k}^{*}\left(F_{*}(f), A\right), k \geqslant 1$, and one gets the required isomorphism.

In order to obtain an axiomatic description of the relative cohomology of groups the category of coefficients will be enlarged giving an extension of the classical relative cohomology theory.

Let $f: G \rightarrow G^{\prime}$ be an epimorphism of groups. Denote $\mathcal{C}_{f}$ the category whose objects are injections $\varphi: A \rightarrow B$ such that $A$ is a $G^{\prime}$-module, $B$ is a $G$-module and $\varphi$ is a morphism of $G$-modules induced by $f$. A morphism $(\alpha, \beta):(\varphi: A \rightarrow B) \rightarrow$ $\left(\varphi^{\prime}: A^{\prime} \rightarrow B^{\prime}\right)$ is a couple of homomorphisms $\alpha: A \rightarrow A^{\prime}, \beta: B \rightarrow B^{\prime}$ preserving the actions and the diagram

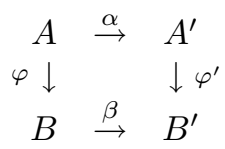

is commutative. The homomorphism $f$ induces a morphism $f^{*}: C^{*}\left(G^{\prime}, A\right) \rightarrow$ $C^{*}(G, B)$ of cochain complexes given by $f^{i}\left(c^{i}\right)=\varphi c^{i} f, i>0, c^{i} \in C^{i}\left(G^{\prime}, A\right)$ and $f^{0}\left(c^{0}\right)=\varphi\left(c^{0}\right)$. It is clear that the homomorphism $f^{i}: C^{i}\left(G^{\prime}, A\right) \rightarrow C^{i}(G, B)$ is injective for all $i \geqslant 0$.

Define the cochain complex $C^{*}(f, \varphi)$ to be the quotient cochain complex Coker $f^{*}$ and its homology $H_{*}\left(\right.$ Coker $\left.f^{*}\right)$ gives the cohomology $H^{*}(f, \varphi)$ of the epimorphism $f$ of groups with coefficients in $\varphi$. From this definition immediately follows a long exact cohomology sequence

$$
\begin{aligned}
0 \rightarrow H^{0}\left(G^{\prime}, A\right) & \rightarrow H^{0}(G, B) \rightarrow H^{0}(f, \varphi) \rightarrow H^{1}\left(G^{\prime}, A\right) \rightarrow \\
H^{1}(G, B) \rightarrow H^{1}(f, \varphi) \rightarrow H^{2}\left(G^{\prime}, A\right) \rightarrow \cdots & \rightarrow H^{n-1}(f, \varphi) \rightarrow \\
H^{n}\left(G^{\prime}, A\right) \rightarrow H^{n}(G, B) \rightarrow H^{n}(f, \varphi) & \rightarrow H^{n+1}\left(G^{\prime}, A\right) \rightarrow \ldots
\end{aligned}
$$

Thus if $G$ and $G^{\prime}$ are free groups, then $H^{n}(f, \varphi)=0$ for $n \geqslant 2$. It is also clear that there is a short exact sequence of cochain complexes

$$
0 \rightarrow C^{*}(f, A) \rightarrow C^{*}(f, \varphi) \rightarrow C^{*}(G, B / A) \rightarrow 0
$$

inducing a long exact cohomology sequence

$$
\begin{gathered}
0 \rightarrow \operatorname{Der}(f, A) \rightarrow Z^{1}(f, \varphi) \rightarrow \operatorname{Der}(G, B / A) \rightarrow H^{2}(f, A) \rightarrow \\
H^{2}(f, \varphi) \rightarrow H^{2}(G, B / A) \rightarrow H^{3}(f, A) \rightarrow \cdots \rightarrow H^{n-1}(G, B / A) \rightarrow \\
H^{n}(f, A) \rightarrow H^{n}(f, \varphi) \rightarrow H^{n}(G, B / A) \rightarrow H^{n+1}(f, A) \rightarrow \cdots .
\end{gathered}
$$


Denote $Z^{k}(f, \varphi)=\operatorname{Ker}\left(C^{k}(f, \varphi) \rightarrow C^{k+1}(f, \varphi)\right), k \geqslant 1$. It is easily seen that if $G^{\prime}$ is a free group, then the sequence $0 \rightarrow Z^{k}\left(G^{\prime}, A\right) \rightarrow Z^{k}(G, B) \rightarrow Z^{k}(f, \varphi) \rightarrow 0$ is exact for $k \geqslant 1$.

Any short exact coefficient sequence $0 \rightarrow \varphi_{1} \rightarrow \varphi \rightarrow \varphi_{2} \rightarrow 0$ in the category $\mathcal{C}_{f}$ (that means both induced short sequences of $G^{\prime}$ and $G$-modules respectively are exact) induces a short exact sequence of cochain complexes $0 \rightarrow C^{*}\left(f, \varphi_{1}\right) \rightarrow$ $C^{*}(f, \varphi) \rightarrow C^{*}\left(f, \varphi_{2}\right) \rightarrow 0$ and therefore a long exact cohomology sequence of the relative cohomology $H^{*}(f,-)$.

Let $(\varphi: A \rightarrow B)$ be an object of the category $\mathcal{C}_{f}$. Let us consider the coinduced $G^{\prime}$-module $A^{*}=\operatorname{Hom}\left(\mathbb{Z}\left[G^{\prime}\right], A\right)$ and the coinduced $G$-module $B^{*}=\operatorname{Hom}(\mathbb{Z}[G], B)$ induced by $A$ and $B$ respectively. Then $f$ induces a well defined homomorphism $\varphi^{*}: A^{*} \rightarrow B^{*}$ and it is easily checked that $\varphi^{*}$ is an object of the category $\mathcal{C}_{f}$ called the coinduced $f$-module induced by $\varphi$. Moreover one has a canonical injection $(\alpha, \beta):(\varphi: A \rightarrow B) \rightarrow\left(\varphi^{*}: A^{*} \rightarrow B^{*}\right)$ induced by the well known injections $A \rightarrow$ $A^{*}$ and $B \rightarrow B^{*}$. The exact cohomology sequence (11) implies that $H^{n}\left(f, \varphi^{*}\right)=0$ for $n \geqslant 1$ and any coinduced $f$-module $\varphi^{*}$. By taking the cokernels one gets a homomorphism $\bar{\varphi}: A^{*} / \operatorname{Im} \alpha \rightarrow B^{*} / \operatorname{Im} \beta$ induced by $\varphi^{*}$.

Proposition 9. The homomorphism $\bar{\varphi}$ is object of the category $\mathcal{C}_{f}$.

Proof. It is sufficient to show that $\bar{\varphi}$ is injective. One has a commutative diagram

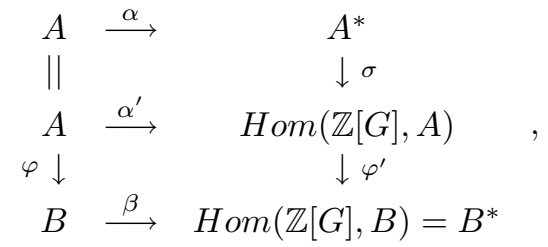

where $\sigma$ is injective and induced by $f$, the group $\operatorname{Hom}(\mathbb{Z}[G], A)$ is the coinduced $G$ module induced by $A$ as a $G$-module and the composite $\varphi^{\prime} \sigma$ is equal to $\varphi^{*}$. Therefore one gets a commutative diagram

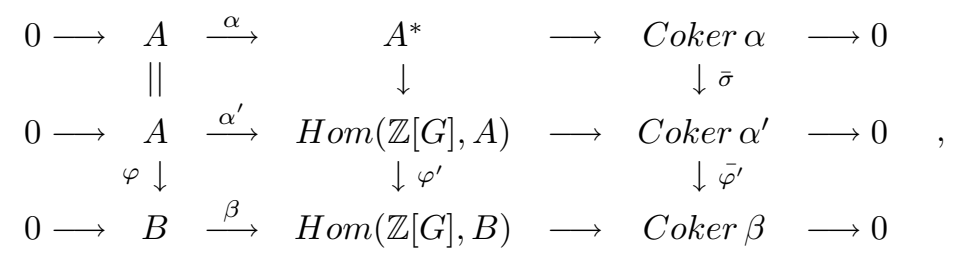

where $\bar{\sigma}$ is injective,since $\sigma$ is. It remains to show that $\bar{\varphi}^{\prime}$ is injective. But this follows from the fact that if $0 \rightarrow A \rightarrow B \rightarrow C \rightarrow 0$ is an exact sequence of abelian groups, then the sequence

$$
0 \rightarrow \operatorname{Hom}(\mathbb{Z}[G], A) \rightarrow \operatorname{Hom}(\mathbb{Z}[G], B) \rightarrow \operatorname{Hom}(\mathbb{Z}[G], C) \rightarrow 0
$$

is also exact, since $\mathbb{Z}[G]$ is a free abelian group.

Corollary 10. For any object $\varphi$ of the category $\mathcal{C}_{f}$ one has a short exact sequence $0 \rightarrow \varphi \rightarrow \varphi * \rightarrow \bar{\varphi} \rightarrow 0$ in the category $\mathcal{C}_{f}$, where $\varphi *$ is a coinduced $f$-module. 
Theorem 11. (i) There is only one (up to equivalence) connected sequence of covariant abelian group valued functors $\left\{T^{n}, \delta^{n}, n \geqslant 0\right\}$ defined on the category $\mathcal{C}_{f}$ and satisfying the following conditions:

1) $T^{0}=Z^{1}(f,-)$.

2) Any short exact sequence $0 \rightarrow \varphi \rightarrow \psi \rightarrow \omega \rightarrow 0$ of the category $\mathcal{C}_{f}$ induces a long exact connected sequence of the functors $T^{n}, n \geqslant 0$.

3) $T^{n}(\varphi)=0$ for $n \geqslant 1$ and any coinduced $f$-module $\varphi$.

(ii) There are natural isomorphisms $R^{n}{ }_{\mathcal{M F}} Z^{k}(f, \varphi) \approx H^{n+k}(f, \varphi), n>0$, for any $f$-module $\varphi$ and $k \geqslant 1$.

Proof. (i) As we have seen the sequence $\left\{Z^{1}(f,-), H^{n}(f,-), \delta^{n}, n>0\right\}$ satisfies conditions 1-3. The proof of the uniqueness is standard.

(ii) Similar to the proof of Theorem 8. In effect, in this case we have also the exact sequence of cotriple derived functors

$$
\begin{gathered}
0 \rightarrow R_{\mathcal{M F}}^{0} Z^{k}\left(G^{\prime}, A\right) \rightarrow R^{0}{ }_{\mathcal{M F}} Z^{k}(G, B) \rightarrow R^{0}{ }_{\mathcal{M F}} Z^{k}(f, \varphi) \rightarrow R^{1} \mathcal{M F}^{k} Z^{k}\left(G^{\prime}, A\right) \rightarrow \\
R^{1}{ }_{\mathcal{M F}} Z^{k}(G, B) \rightarrow R^{1}{ }_{\mathcal{M F}} Z^{k}(f, \varphi) \rightarrow R^{2}{ }_{\mathcal{M F}} Z^{k}\left(G^{\prime}, A\right) \rightarrow \ldots \rightarrow \\
R^{n-1}{ }_{\mathcal{M F}} Z^{k}(f, \varphi) \rightarrow R^{n}{ }_{\mathcal{M F}} Z^{k}\left(G^{\prime}, A\right) \rightarrow R^{n}{ }_{\mathcal{M F}} Z^{k}(G, B) \rightarrow \\
R^{n}{ }_{\mathcal{M F}} Z^{k}(f, \varphi) \rightarrow R^{n+1}{ }_{\mathcal{M F}} Z^{k}\left(G^{\prime}, A\right) \rightarrow \cdots
\end{gathered}
$$

for $f: G \rightarrow G^{\prime}$ and $\varphi: A \rightarrow B$. The 0-derived functors could be replaced by $Z^{k}\left(G^{\prime}, A\right), Z^{k}(G, B)$ and $Z^{k}(f, \varphi)$ respectively. If we denote $C_{k}^{n}(f, \varphi)=C^{n+k}(f, \varphi)$, for all $n \geqslant 0, k \geqslant 1$, it is easily checked that $H_{0}\left(C_{k}^{*}(f, \varphi)\right)=Z^{k}(f, \varphi)$ and if the groups $G$ and $G^{\prime}$ are free groups, then $H_{n}\left(C_{k}^{*}(f, \varphi)\right)=0$ for $n \geqslant 1$. On the other hand, the short exact sequence of cochain complexes

$$
0 \rightarrow C_{k}^{j}\left(F_{*}\left(G^{\prime}\right), A\right) \rightarrow C_{k}^{j}\left(F_{*}(G), B\right) \rightarrow C_{k}^{j}\left((F M)_{*}(f), \varphi\right) \rightarrow 0, j \geqslant 0,
$$

gives the equalities $H_{n}\left(C_{k}^{j}\left((M F)_{*}(f), \varphi\right)\right)=0, n>0$, and $H_{0}\left(C_{k}^{j}\left((M F)_{*}(f), \varphi\right)\right)=$ $C_{k}^{j}(f, \varphi)$ for $j \geqslant 0$ and $k \geqslant 1$.

It remains to use the bicomplex $C_{k}^{*}\left((M F)_{*}(f), \varphi\right)$ to get the required isomorphisms via the homology of the total cochain complex $\operatorname{Tot} C_{k}^{*}\left((M F)_{*}(f), \varphi\right), k \geqslant 1$, where $(M F)_{*}(f) \rightarrow f$ is the cotriple resolution of $f$ with respect to the cotriple $\mathcal{M F}$.

It is clear if $f: F \rightarrow G$ is a surjective group homomorphism with $F$ a free group and $A$ is a $G^{\prime}$-module, then for $k \geqslant 1$ one has an exact sequence

$$
0 \rightarrow Z^{k}(G, A) \rightarrow Z^{k}(F, A) \rightarrow Z^{k}(f, A) \rightarrow H^{k+1}(G, A) \rightarrow 0 .
$$

Proposition 12. For any surjective group homomorphism $f: G \rightarrow G^{\prime}$ and a $G^{\prime}$ module $A$ there is an exact sequence

$$
0 \rightarrow Z^{1}(f, A) \rightarrow Z^{1}\left(f, 1_{A}^{*}\right) \rightarrow Z^{1}\left(f, \overline{1}_{A}\right) \rightarrow H^{2}(f, A) \rightarrow 0
$$

and $H^{k}\left(f, \overline{1}_{A}\right) \approx H^{k+1}(f, A), k \geqslant 2$.

Proof. By Corollary 10 one can consider the short exact coefficient sequence

$$
0 \rightarrow 1_{A} \rightarrow 1_{A}^{*} \rightarrow \overline{1}_{A} \rightarrow 0
$$


and apply Theorem 11 .

Notice that the relative cohomology group $H^{n}(f, A)$ coincides with the relative cohomology group $H^{n+1}\left(G^{\prime}, G, A\right), n \geqslant 2$, defined in [17], where a different interpretation of the cohomology group $H^{3}\left(G^{\prime}, G, A\right)$ is given in terms of relative extensions of $\left(G^{\prime}, G\right)$.

Remark 13. It is obvious that by (ii) of Theorem 11 we recover Theorem 8 for $\varphi=1_{A}: A \rightarrow A$. To get results similar to Theorem 11 for the homology of surjective homomorphisms it is necessary to consider as coefficients the category of surjections $f: B \rightarrow A$ of $G$-modules with $A$ a $G^{\prime}$-module and the proof is similar. The case $k=1$ for Theorem 8 has been also considered in [19].

Now the description of $H^{2}(f, A)$ given in Proposition 12 will be extended to higher relative cohomology groups.

Let $f: G \rightarrow G^{\prime}$ be a surjective group homomorphism and $\varphi: A \rightarrow B$ be an object of the category $\mathcal{C}_{f}$. Consider pairs $E_{k}=\left(e_{k}, \psi_{k}\right)$ consisting of an exact coefficient sequence $e_{k}=0 \rightarrow \varphi \rightarrow \varphi_{1} \rightarrow \cdots \rightarrow \varphi_{k+1} \rightarrow 0$ and an element $\psi_{k}$ of the group $Z^{1}\left(f, \varphi_{k+1}\right), k \geqslant 1$. It will be said that a pair $E_{k}=\left(e_{k}, \psi_{k}\right)$ is similar to a pair $E_{k}^{\prime}=\left(e_{k}^{\prime}, \psi_{k}^{\prime}\right)$, if there is a morphism $\alpha=\left(1 \varphi, \alpha_{1}, \cdots, \alpha_{k+1}\right): e_{k} \rightarrow e_{k}^{\prime}$ and there exists an element $\eta_{k-1}^{\prime}$ of the group $Z^{1}\left(f, \varphi_{k}^{\prime}\right)$ such that $\sigma_{k}^{\prime}\left(\eta_{k-1}^{\prime}\right)=\alpha_{k+1}\left(\psi_{k}\right)-\psi_{k}^{\prime}$, where $\sigma_{k}^{\prime}: Z^{1}\left(f, \varphi_{k}^{\prime}\right) \rightarrow Z^{1}\left(f, \varphi_{k+1}^{\prime}\right)$ is induced by the morphism $\varphi_{k}^{\prime} \rightarrow \varphi_{k+1}^{\prime}$ of the sequence $e_{k}^{\prime}$. Denote by $E^{k}(f, \varphi)$ the set of equivalence classes, $k \geqslant 1$, with respect to the equivalence relation generated by the relation of similar pairs. We shall introduce a "Baer sum" on the set $E^{k}(f, \varphi)$ as follows.

Let $e_{1}=0 \rightarrow \varphi \rightarrow \varphi_{1} \rightarrow \varphi_{2} \rightarrow 0$ be a short exact sequence and $(\alpha, \beta): \varphi \rightarrow \varphi^{\prime}$ a morphism of the category $\mathcal{C}_{f}$, where $\varphi: A \rightarrow B, \varphi_{i}: A_{i} \rightarrow B_{i}, i=1,2$. Denote by $E_{1}$ the corresponding short exact sequence $0 \rightarrow A \rightarrow A_{1} \rightarrow A_{2} \rightarrow 0$ of $G^{\prime}$ modules and by $E_{2}$ the short exact sequence $0 \rightarrow B \rightarrow B_{1} \rightarrow B_{2} \rightarrow 0$ of $G$-modules respectively. One gets short exact sequences $E_{1}^{\prime}=E\left(A_{2}, \alpha\right)=0 \rightarrow A^{\prime} \rightarrow A_{1}^{\prime} \rightarrow$ $A_{2}^{\prime} \rightarrow 0$ and $E_{2}^{\prime}=E\left(B_{2}, \beta\right)=0 \rightarrow B^{\prime} \rightarrow B_{1}^{\prime} \rightarrow B_{2}^{\prime} \rightarrow 0$ of $G^{\prime}$-modules and $G$ modules respectively, where $E$ denotes the extension functor. It is easy to see that homomorphisms $\varphi_{i}^{\prime}: A_{i}^{\prime} \rightarrow B_{i}^{\prime}$, induced by $\varphi_{i}, i=1,2$, respectively are objects of the category $\mathcal{C}_{f}$ and therefore we obtain a short exact sequence $e^{\prime}=0 \rightarrow \varphi^{\prime} \rightarrow$ $\varphi_{1}^{\prime} \rightarrow \varphi_{2}^{\prime} \rightarrow 0$ in the category $\mathcal{C}_{f}$ which we denote $e_{1}^{\prime}=E\left(e_{1},(\alpha, \beta)\right)$. The definition of $e_{k}^{\prime}=E\left(e_{k},(\alpha, \beta)\right)$ for $e_{k}=0 \rightarrow \varphi \rightarrow \varphi_{1} \rightarrow \cdots \rightarrow \varphi_{k} \rightarrow \varphi_{k+1} \rightarrow 0$ is extended in the usual way for $k>1$.

For $\left[E_{k}\right],\left[E_{k}^{\prime}\right] \in E^{k}(f, \varphi), E_{k}=\left(e_{k}, \psi_{k}\right), E_{k}^{\prime}=\left(e_{k}^{\prime}, \psi_{k}^{\prime}\right)$, define $\left[E_{k}\right]+\left[E_{k}^{\prime}\right]=$ $\left[\left(E\left(e_{k}+e_{k}^{\prime}, \nabla\right), \varphi_{k+1}^{\prime}\left(\psi_{k}+\psi_{k}^{\prime}\right)\right)\right]$, where the morphism $\nabla: \varphi+\varphi \rightarrow \varphi$ is induced by $1_{\varphi}$. It is easy to check that this sum is correctly defined and one gets an abelian group structure on $E^{k}(f, \varphi)$.

Theorem 14. For $k \geqslant 1$ there is a natural isomorphism $\vartheta_{k}: E^{k}(f, \varphi) \rightarrow H^{k+1}(f, \varphi)$.

Proof. At first consider the case $k=1$. The map $\vartheta_{1}: E^{1}(f, \varphi) \rightarrow H^{2}(f, \varphi)$ is given by $\vartheta_{1}\left(\left[\left(e_{1}, \psi_{1}\right)\right]\right)=\delta^{1}\left(\psi_{1}\right)$, where $e_{1}=0 \rightarrow \varphi \rightarrow \varphi_{1} \rightarrow \varphi_{2} \rightarrow 0, \psi_{1} \in Z^{1}\left(f, \varphi_{2}\right)$ and $\delta^{1}$ is the connecting homomorphism of the long exact cohomology sequence induced by the exact coefficient sequence $e_{1}$. 
To define the converse map $\vartheta_{1}^{\prime}: H^{2}(f, \varphi) \rightarrow E^{1}(f, \varphi)$ use the short exact sequence $e=0 \rightarrow \varphi \rightarrow \varphi^{*} \rightarrow \bar{\varphi} \rightarrow 0$ implying an epimorphism $\delta^{1}: Z^{1}(f, \bar{\varphi}) \rightarrow H^{2}(f, \varphi)$ and define $\vartheta_{1}^{\prime}(x)=[(e, \psi)]$, where $\delta^{1}(\psi)=x$ for $x \in H^{2}(f, \varphi)$.

Let $k=2$ and $\left[\left(e_{2}, \psi_{2}\right)\right] \in E^{2}(f, \varphi)$, where $e_{2}=0 \rightarrow \varphi \rightarrow \varphi_{1} \rightarrow \varphi_{2} \rightarrow \varphi_{3} \rightarrow$ 0 and $\psi_{2} \in Z^{1}\left(f, \varphi_{3}\right)$. The exact sequence $e_{2}$ induces two short exact sequences $e_{1}: 0 \rightarrow \varphi \rightarrow \varphi_{1} \rightarrow \operatorname{Im} \varphi_{1} \rightarrow 0$ and $e_{1}^{\prime}: 0 \rightarrow \operatorname{Im} \varphi_{1} \rightarrow \varphi_{2} \rightarrow \varphi_{3} \rightarrow 0$. Define the map $\vartheta_{2}: E^{2}(f, \varphi) \rightarrow H^{3}(f, \varphi)$ by $\vartheta_{2}\left(\left[\left(e_{2}, \psi_{2}\right)\right]\right)=\delta_{e_{1}}^{2} \delta_{e_{1}^{\prime}}^{1}\left(\psi_{2}\right)$. The map $\vartheta_{2}^{\prime}$ : $H^{3}(f, \varphi) \rightarrow E^{2}(f, \varphi)$ is defined as follows. Splice the short exact sequences $e_{1}$ : $0 \rightarrow \varphi \rightarrow \varphi * \rightarrow \bar{\varphi} \rightarrow 0$ and $e_{1}^{\prime}: 0 \rightarrow \bar{\varphi} \rightarrow \bar{\varphi} * \rightarrow \overline{\bar{\varphi}} \rightarrow 0$ to get the exact sequence $e_{2}=0 \rightarrow \varphi \rightarrow \varphi * \rightarrow \bar{\varphi} * \rightarrow \overline{\bar{\varphi}} \rightarrow 0$. Clearly $\delta_{e_{1}}^{2}: H^{2}(f, \bar{\varphi}) \rightarrow H^{3}(f, \varphi)$ is an isomorphism and $\delta_{e_{1}^{\prime}}^{1}: Z^{1}(f, \bar{\varphi}) \rightarrow H^{2}(f, \bar{\varphi})$ is surjective. Then $\vartheta_{2}^{\prime}$ is given by $\vartheta_{2}^{\prime}(x)=\left[\left(e, \psi_{2}\right)\right]$, where $\delta_{e_{1}^{\prime}}^{1}\left(\psi_{2}\right)=\left(\delta_{e_{1}}^{2}(x)\right)^{-1}$ for $x \in H^{3}(f, \varphi)$.

For $k>2$ the construction of the maps $\vartheta_{k}$ and $\vartheta_{k}^{\prime}$ are similar to the case $k=2$. It is routine to show that these maps for all $k \geqslant 1$ are correctly defined homomorphisms, both composites $\vartheta_{k} \vartheta_{k}^{\prime}, \vartheta_{k}^{\prime} \vartheta_{k}$ are identity maps, and left to the reader.

\section{Cohomology of groups with operators}

Recently in [7] a cohomology theory $H_{\Gamma}^{*}(G, A)$ of a group $G$ with operators was defined, when a separate group $\Gamma$ acts on $G$, with coefficients in a $\Gamma$-equivariant $G$ module. This cohomology theory which will be called shortly equivariant cohomology of groups was motivated by the graded categorical groups classification problem suggested by Frohlich and Wall [14] and solved in [8] by using the equivariant group cohomology $H_{\Gamma}^{3}(G, A)$.

It was shown that $H_{\Gamma}^{*}(G, A)$ is a cotriple cohomology as the non-abelian derived functors of the group $\operatorname{Der}_{\Gamma}(G, A)$ of $\Gamma$-equivariant derivations from $G$ to $A$ with respect to the free cotriple defined in the category of $\Gamma$-groups acting on $A$.

Let $G$ and $\Gamma$ be arbitrary groups, and $G$ a $\Gamma$-group. A $\Gamma$-module $A$ is called a $\Gamma$-equivariant $G$-module, if it is a $G$-module such that ${ }^{y}\left({ }^{x} a\right)={ }^{\left({ }^{y} x\right)}\left({ }^{y} a\right)$ for $y \in \Gamma$, $x \in G, a \in A$. A $\Gamma$-equivariant derivation (or crossed $\Gamma$-homomorphism) from $G$ into the $G$-module $A$ is a map $f: G \rightarrow A$ satisfying the conditions $f(x y)=f(x)+{ }^{x} f(y)$ for $x, y \in G$ and $f\left({ }^{y} x\right)={ }^{y} f(x)$ for $y \in \Gamma, x \in G$.

The cochain complex $C_{\Gamma}^{*}(G, A)$ defining the equivariant group cohomology $H_{\Gamma}^{*}(G, A)$ is given by

$$
0 \rightarrow C_{\Gamma}^{0}(G, A) \rightarrow C_{\Gamma}^{1}(G, A) \rightarrow C_{\Gamma}^{2}(G, A) \rightarrow C_{\Gamma}^{3}(G, A) \rightarrow \cdots \rightarrow C_{\Gamma}^{n}(G, A) \rightarrow \cdots,
$$

where the elements of $C_{\Gamma}^{n}(G, A)$ are maps

$$
f: \cup_{p+q=n-1} G^{p+1} \times \Gamma^{q} \rightarrow A, n \geqslant 1, C_{\Gamma}^{0}(G, A)=0,
$$

and the coboundary operator $\delta^{n}: C^{n} \Gamma(G, A) \rightarrow C_{\Gamma}^{n+1}(G, A)$ is defined by the 
formula

$$
\begin{gathered}
\delta^{n}(f)\left(x_{1}, \ldots, x_{p+1}, \sigma_{1}, \ldots, \sigma_{q}\right)={ }^{\sigma_{1}} f\left(x_{1}, \ldots, x_{p+1}, \sigma_{2}, \ldots, \sigma_{q}\right)+ \\
\sum(-1)^{i} f\left(x_{1}, \ldots, x_{p+1}, \sigma_{1}, \ldots, \sigma_{i} \sigma_{i+1}, \ldots, \sigma_{q}\right)+ \\
(-1)^{q} f\left({ }^{\sigma_{q}} x_{1}, \ldots,{ }^{\sigma_{q}} x_{p+1}, \sigma_{1}, \ldots, \sigma_{q-1}\right)+ \\
(-1)^{q}\left[{ }^{\left(\sigma_{1} \cdots \sigma_{q}\right.} x_{1} f\left(x_{2}, \ldots, x_{p+1}, \sigma_{1}, \ldots, \sigma_{q}\right)+\right. \\
\sum(-1)^{j} f\left(x_{1}, \ldots, x_{j} x_{j+1}, \cdots, x_{p+1}, \cdots, \sigma_{1}, \ldots, \sigma_{q}\right)+ \\
\left.(-1)^{p+1} f\left(x_{1}, \ldots, x_{p}, \sigma_{1}, \ldots, \sigma_{q}\right)\right]
\end{gathered}
$$

with $x_{1}, \ldots, x_{p+1} \in G, \sigma_{1}, \ldots, \sigma_{q} \in \Gamma$ and $p+q=n$. Then $H_{\Gamma}^{n}(G, A)=H_{n} C_{\Gamma}^{*}(G, A)$, $n \geqslant 0$. Thus $H_{\Gamma}^{0}(G, A)=0$ and $H_{\Gamma}^{1}(G, A)=\operatorname{Der}_{\Gamma}(G, A)$.

Denote $Z_{\Gamma}^{k}(G, A)=\operatorname{Ker} \delta^{k}, k \geqslant 1$. One gets contravariant functors $Z_{\Gamma}^{k}(-, A)$ : $\mathcal{G}_{\Gamma, A} \rightarrow \mathfrak{A} \mathfrak{b}, n \geqslant 1$, from the category of $\Gamma$-groups acting on $A$ to the category of abelian groups. Clearly $Z_{\Gamma}^{1}(G, A)=\operatorname{Der}_{\Gamma}(G, A)$. In [7] is defined the free cotriple $\mathcal{F}_{\Gamma}=\left(F_{\Gamma}, \epsilon, \delta\right)$ in the category $\mathcal{G}_{\Gamma}$ given by $F_{\Gamma}(G)$ which is the free group generated by the set $G \times \Gamma$ with the action ${ }^{\sigma}(g, \gamma)=(g, \sigma \gamma)$, the homomorphism $\epsilon: F_{\Gamma}(G) \rightarrow G$ is given by $\epsilon(g, \gamma)={ }^{\gamma} g$ and the homomorphism $\delta: F_{\Gamma}(G) \rightarrow F_{\Gamma}^{2}(G)$ is defined by $\delta(g, \gamma)={ }^{\delta}((g, 1), \gamma)$.

Let us consider now the cotriple derived functors $R_{\mathcal{F}_{\Gamma}}^{n} Z_{\Gamma}^{k}(-, A), n \geqslant 0, k \geqslant 1$, of the contravariant functors $Z_{\Gamma}^{k}(-, A)$ with respect to the free cotriple $\mathcal{F}_{\Gamma}$.

Theorem 15. There is a natural isomorphism

$$
R_{\mathcal{F}_{\Gamma}}^{n} Z_{\Gamma}^{k}(G, A) \approx H_{\Gamma}^{n+k}(G, A)
$$

for all $n>0, k>0$ and any $\Gamma$-equivariant $G$-module $A$.

Proof. The case $k=1$ is proved in [7] (see Theorem 1.6). It follows that $H_{\Gamma}^{n}(G, A)=$ 0 for $n \geqslant 2$, if $G$ belongs to the projective class $\mathcal{P}_{\Gamma}$ generated by the free cotriple $\mathcal{F}_{\Gamma}$. For $k>1$ the proof is similar to the case of classical cohomology of groups (see Theorem 1) and we will shortly repeat it.

One has a functorial isomorphism $R_{\mathcal{F}_{\Gamma}}^{0} Z_{\Gamma}^{k}(-, A) \approx Z_{\Gamma}^{k}(-, A), k>0$. Therefore for the equivariant group cohomology $H_{\Gamma}^{*}(G, A)$ one gets exact sequences like (2) and (4). In [6] it is shown that $H_{\Gamma}^{n}(G, A)=0$ for $n \geqslant 2$, if $A$ is an injective $\Gamma$-equivariant $G$-module. Since any short exact sequence of $\Gamma$-equivariant $G$-modules provides a long exact equivariant cohomology sequence,to complete the proof it remains to show that $R_{\mathcal{F} \Gamma}^{n} Z_{\Gamma}^{k}(G, A)=0$ for $n, k>1$, if $A$ is an injective $\Gamma$-equivariant $G$ module.

Let $F_{*}(G) \rightarrow G$ be the free cotriple resolution of $G$ with respect to $\mathcal{F}_{\Gamma}$. By applying the defining cochain complex (12) to this resolution we obtain a sequence of abelian cosimplicial groups

$$
0 \rightarrow C_{\Gamma}^{1}\left(F_{*}(G), A\right) \rightarrow C_{\Gamma}^{2}\left(F_{*}(G), A\right) \rightarrow C_{\Gamma}^{3}\left(F_{*}(G), A\right) \rightarrow \cdots \rightarrow C_{\Gamma}^{n}\left(F_{*}(G), A\right) \rightarrow \cdots
$$

which is exact for any $\Gamma$-equivariant $G$-module except at the first term. Assume now that $A$ is an injective $\Gamma$-equivariant $G$-module. Then the short exact sequence 
of abelian cosimplicial groups

$$
0 \rightarrow \underset{\Gamma}{\operatorname{Der}}\left(F_{*}(G), A\right) \rightarrow C_{\Gamma}^{1}\left(F_{*}(G), A\right) \rightarrow \operatorname{Im} \delta_{\Gamma}^{1} \rightarrow 0
$$

implies $H_{n}\left(\operatorname{Im} \delta_{\Gamma}^{1}\right)=0$ for $n>0$, by taking into account that $H_{n}\left(C_{\Gamma}^{i}\left(F_{*}(G), A\right)\right)=0$ for all $n, i>0$ and any $\Gamma$-equivariant $G$-module, since the augmented simplicial group $F_{*}(G) \rightarrow G$ is left contractible as augmented simplicial set. But $\operatorname{Im} \delta_{\Gamma}^{1}=$ $\operatorname{Ker} \delta_{\Gamma}^{2}$ providing $H_{n}\left(Z_{\Gamma}^{2}\left(F_{*}(G), A\right)\right)=0, n>0$. By using the exactness of $(13)$ and the induction on $k$ finally one gets $H_{n}\left(Z_{\Gamma}^{k}\left(F_{*}(G), A\right)\right)=0$ for all $n>0, k \geqslant 1$.

\section{5. (Co)homology of associative algebras}

We consider the category $\mathcal{A}$ of associative algebras over a unital commutative ring $k$. Let $A$ be an associative $k$-algebra, $M$ a bimodule over $A$ and take the standard cochain complex

$$
0 \rightarrow C^{0}(A, M) \stackrel{\delta^{0}}{\rightarrow} C^{1}(A, M) \stackrel{\delta^{1}}{\rightarrow} C^{2}(A, M) \stackrel{\delta^{2}}{\rightarrow} C^{3}(A, M) \stackrel{\delta^{3}}{\rightarrow} \cdots
$$

and the standard chain complex

$$
\cdots \stackrel{\partial_{4}}{\rightarrow} C_{3}(A, M) \stackrel{\partial_{3}}{\rightarrow} C_{2}(A, M) \stackrel{\partial_{2}}{\rightarrow} C_{1}(A, M) \stackrel{\partial_{1}}{\rightarrow} C_{0}(A, M) \rightarrow 0
$$

where $C_{n}(A, M)=M \otimes A^{\otimes n}, \partial_{n}=\sum(-1)^{i} d^{i}$ and $C^{n}(A, M)=H_{k}\left(A^{\otimes n}, M\right)$, $\otimes=\otimes_{k}$, with

$$
\begin{aligned}
& d_{0}\left(m, a_{1}, \ldots, a_{n}\right)=\left(m a_{1}, a_{2}, \ldots, a_{n}\right), \\
& d_{i}\left(m, a_{1}, \ldots, a_{n}\right)=\left(m, a_{1}, \ldots, a_{i} a_{i+1}, \ldots, a_{n}\right) \text { for } 1 \leqslant i<n, \\
& d_{n}\left(m, a_{1}, \ldots, a_{n}\right)=\left(a_{n} m, a_{1}, \ldots, a_{n-1}\right),
\end{aligned}
$$

which give respectively the Hochschild cohomology $H H^{*}(A, M)$ and homology $H H_{*}(A, M)$ of $A$ with coefficients in $M$ (see [18]).

Denote $A^{e}=A \otimes_{k} A^{o p}$ and $J A=\operatorname{Ker}\left(A^{e} \rightarrow A\right)$ with $\epsilon(a \otimes b)=a b$. It is well known that for $A$ projective over $k$ one has the standard $A^{e}$-projective resolution of $A$ :

$$
\cdots \rightarrow A^{\otimes n+1} \stackrel{b_{n-1}}{\longrightarrow} A^{\otimes n} \stackrel{b_{n-2}}{\longrightarrow} \cdots \stackrel{b_{1}}{\rightarrow} A^{\otimes 2} \stackrel{b_{0}}{\longrightarrow} A,
$$

where $b_{n-2}=\sum_{i=0}^{n-1}(-1)^{i} d_{i}$ for $n>2$ and $b_{0}(a \otimes b)=a b$. Clearly $K e r b_{0}=J A$ as $A^{e}$-modules. Denote $Y_{k}(A)=$ Coker $_{b}$ for $k \geqslant 1$.

Clearly the group of derivations $\operatorname{Der}(A, M)$ is isomorphic to the kernel of $\delta^{1}$ and Coker $\partial^{2}$ is isomorphic to $J A \otimes_{A^{e}} M$. We introduce the following notations:

$$
Y_{k}(A, M)=Y_{k}(A) \otimes_{A^{e}} M, k>0, \text { and } Z^{k}(A, M)=\operatorname{Ker} \delta^{k}, k>0 .
$$

Thus $Y_{1}(A, M) \approx J A \otimes_{A^{e}} M$ and $Z^{1}(A, M) \approx \operatorname{Der}(A, M)$. It is obvious that $Y_{k}(-, M)$ and $Z^{k}(-, M), k>0$, are respectively covariant and contravariant functors from the category $\mathcal{A}$ to the category of abelian groups.

Let $\mathcal{F}$ be the free cotriple defined for any associative $k$-algebra $A$ by taking the free associative $k$-algebra $F(A)$ over the underlying set $A$. Let us consider the cotriple (left) derived functors $L_{n}^{\mathcal{F}} Y_{k}(-, M)$ of the functors $Y_{k}(-, M)$ for all $k>$ 
$0, n \geqslant 0$, and the cotriple (right) derived functors $R_{\mathcal{F}}^{n} Z^{k}(-, M)$ of the functors $Z^{k}(-, M)$ for all $k>0, n \geqslant 0$, with respect to the free cotriple $\mathcal{F}$.

Theorem 16. There are natural isomorphisms

$$
\begin{aligned}
& L_{n}^{\mathcal{F}} Y_{k}(A, M) \approx H H_{n+k}(A, M), \\
& R_{\mathcal{F}}^{n} Z^{k}(A, M) \approx H H^{n+k}(A, M)
\end{aligned}
$$

for $n, k>0$ and any associative $k$-algebra A projective over $k$.

These isomorphisms are well known for $n>0$ and $k=1$ (see [2]). For $k>1$ the proof is similar to the proof of Theorem 1 and will be omitted.

\section{Cohomology of crossed modules}

Let $\partial: T \rightarrow G$ be a crossed module and $\mathcal{G}$ the free cotriple in the category of crossed modules (see [5]). Then $\mathcal{G}(\partial)$ is the inclusion $\bar{F}(T \times G) \hookrightarrow F(T \times G) *$ $F(T \times G)$, where $\bar{F}(T \times G)=\operatorname{Ker}(F(T \times G) * F(T \times G) \rightarrow F(T \times G)), F(T \times G)$ is the free group generated by the set $T \times G$ and $p_{2} u_{1}=0, p_{2} u_{2}=1_{F(T \times G)}$ with $u_{1}, u_{2}: F(T, G) \hookrightarrow F(T \times G) * F(T \times G)$ natural inclusions. Denote $\mathcal{G}_{i}(\partial)=T_{i} \hookrightarrow G_{i}$ and $\tau_{i}: G_{i} \rightarrow G_{i} / T_{i}$, where $G_{i}$ and $G_{i} / T_{i}$ are free groups, $i \geqslant 0$, with $\mathcal{G}_{0}(\partial)=\mathcal{G}(\partial)$ and $\mathcal{G}_{i}=\mathcal{G}\left(\mathcal{G}_{i-1}\right), i \geqslant 1$.

Let us consider the category $\mathcal{G}_{1}$ of triples $\left(l_{0}, l_{1}, s\right)$ consisting of group homomorphisms $l_{i}: L \rightarrow G, i=0,1$, and $s: G \rightarrow L$ such that $l_{i} s=1_{G}, i=0,1$. Morphisms of the category $\mathcal{G}_{1}$ are couples $(\alpha, \beta):\left(l_{0}, l_{1}, s\right) \rightarrow\left(l_{0}^{\prime}, l_{1}^{\prime}, s^{\prime}\right)$ of group homomorphisms $\alpha: L \rightarrow L^{\prime}$ and $\beta: G \rightarrow G^{\prime}$ such that $\beta l_{i}=l_{i}^{\prime} \alpha, i=0,1$ and $\alpha s=s^{\prime} \beta$. There are two important full subcategories $\mathcal{G}_{2}$ and $\mathcal{G}_{3}$ whose objects $\left(l_{0}, l_{1}, s\right)$ satisfy the conditions $\left[K \operatorname{Ker} l_{0}, K e r l_{1}\right]=1$ and $\operatorname{Ker} l_{0} \cap K \operatorname{Ker} l_{1}=1$ respectively. Clearly one has the inclusions $\mathcal{G}_{1} \supset \mathcal{G}_{2} \supset \mathcal{G}_{3}$. It is well known that these categories are equivalent in the same order to the categories $\mathcal{P C M} \supset \mathcal{C M} \supset \mathcal{I} \mathcal{J}$ of precrossed modules, crossed modules and injective (aspherical) crossed modules respectively. It is obvious that the latter is equivalent to the category $\mathcal{M G}$ of surjective group homomorphisms. The equivalence is established as follows: to any precrossed module of groups $\partial: T \rightarrow G$ corresponds the triple $\left(d_{0}, d_{1}, s\right)_{\partial}$ consisting of $d_{0}, d_{1}: T \bowtie G \rightarrow G$ and $s: G \rightarrow T \bowtie G$, where $T \bowtie G$ is the semidirect product of $T$ by $G, d_{0}(t, g)=g, d_{1}(t, g)=\partial(t) \cdot g$ and $s(g)=(1, g)$.

The notion of the group of derivations in an abelian group $A$ for surjective group homomorphisms (see definition 7 ), or equivalently for objects of the category $\mathcal{I} \mathcal{J}$, has been extended in [19] for crossed modules by using the above defined equivalence of the category $\mathcal{C M}$ with the category $\mathcal{G}_{2}$ and by considering the category of crossed modules acting on an abelian group $A$.

In order to define the cohomology of crossed modules, as for the case of the relative cohomology of groups we want to enlarge the category of coefficients. Let $\partial: T \rightarrow G$ be a precrossed module. Denote $C_{\partial}$ the category of injections $\varphi: A \rightarrow B$ of abelian groups such that $A$ is a $(G / \operatorname{Im} \partial)$-module, $G$ acts on $B$ and $\varphi$ is a $G$ module, $G$ acting on $A$ via the canonical homomorphism $G \rightarrow G / \operatorname{Im} \partial$. In this case it will be said that the precrossed module $\partial$ acts on $\varphi$. The category $C_{\partial}$ was defined 
above, when $\partial$ is injective and $G$ acts on $T$ by conjugation or equivalently for the canonical surjective homomorphism $G \rightarrow G / \operatorname{Im} \partial$.

Let $\partial: T \rightarrow G$ be a crossed module acting on an injection $\varphi: A \rightarrow B$ of abelian groups. This action induces actions of $\mathcal{G}_{i}(\partial)$ and $\tau_{i}$ on $\varphi$ for all $i \geqslant 0$. So the standard cochain complexes $C^{*}\left(\tau_{i}, \varphi\right), i \geqslant 0$, are well defined. Take new cochain complexes $\bar{C}^{*}\left(\tau_{i}, \varphi\right), i \geqslant 0$, getting from $C^{*}\left(\tau_{i}, \varphi\right)$ by dimension shifting, i.e. $\bar{C}^{n}\left(\tau_{i}, \varphi\right)=C^{n+1}\left(\tau_{i}, \varphi\right)$ for $n, i \geqslant 0$. Consider now the bicomplex $\bar{C}^{*}\left(\tau_{*}, \varphi\right)$ and its total cochain complex $\operatorname{Tot} \bar{C}^{*}\left(\tau_{*}, \varphi\right)$ constructed for the cotriple resolution $\mathcal{G}_{*}(\partial) \rightarrow \partial$ of the crossed module $\partial$ with respect to the free cotriple $\mathcal{G}$. Denote by $Z^{1}(\partial, \varphi)$ the kernel of the differential $\delta^{1}$ of the cochain complex $\operatorname{Tot} C^{*}\left(\tau_{*}, \varphi\right)$, $k \geqslant 0$.

Definition 17. The cohomology groups of the crossed module $(\partial: T \rightarrow G)$ with coefficients in $(\varphi: A \rightarrow B)$ is given by $H^{n}(\partial, \varphi)=H_{n}\left(\operatorname{Tot} \bar{C}^{*}\left(\tau_{*}, \varphi\right)\right)$ for $n \geqslant 0$.

It is easily checked that these cohomology groups are independent of the projective resolution of the crossed module $\partial$ with respect to the projective class induced by the free cotriple $\mathcal{G}$.

Theorem 18. Let $\partial: T \rightarrow G$ be a crossed module. Then

(i) Any short exact sequence of coefficients $0 \rightarrow \varphi_{1} \rightarrow \varphi \rightarrow \varphi_{2} \rightarrow 0$ induces a long exact cohomology sequence

$$
\begin{gathered}
0 \rightarrow H^{0}\left(\partial, \varphi_{1}\right) \rightarrow H^{0}(\partial, \varphi) \rightarrow H^{0}\left(\partial, \varphi_{2}\right) \rightarrow H^{1}\left(\partial, \varphi_{1}\right) \rightarrow \cdots \rightarrow \\
H^{n-1}\left(\partial, \varphi_{2}\right) \rightarrow H^{n}\left(\partial, \varphi_{1}\right) \rightarrow H^{n}(\partial, \varphi) \rightarrow \\
H^{n}\left(\partial, \varphi_{2}\right) \rightarrow H^{n+1}\left(\partial, \varphi_{1}\right) \rightarrow \cdots
\end{gathered}
$$

(ii) If $\partial$ acts on $(\varphi: A \rightarrow B)$, there is a long exact cohomology sequence

$$
\begin{gathered}
0 \rightarrow \operatorname{Der}(\partial, A) \rightarrow Z^{1}(\partial, \varphi) \rightarrow \operatorname{Der}(G, B / \operatorname{Im} \varphi) \rightarrow D^{1}(\partial, A) \rightarrow H^{1}(\partial, \varphi) \rightarrow \\
H^{2}(G, B / \operatorname{Im} \varphi) \rightarrow D^{2}(\partial, A) \rightarrow H^{2}(\partial, \varphi) \rightarrow \cdots \rightarrow H^{n}(G, B / \operatorname{Im} \varphi) \rightarrow \\
D^{n}(\partial, A) \rightarrow H^{n}(\partial, \varphi) \rightarrow H^{n+1}(G, B / \operatorname{Im} \varphi) \rightarrow D^{n+1}(\partial, A) \rightarrow \cdots,
\end{gathered}
$$

there is an isomorphism

$$
H^{n}\left(\partial, A \rightarrow A^{*}\right) \approx H^{n+2}(B(T, G, \partial), A), \quad n>0,
$$

and an exact sequence

$$
\begin{gathered}
0 \rightarrow \operatorname{Der}(G / \operatorname{Im} \partial, A) \rightarrow \operatorname{Der}\left(G, A^{*}\right) \rightarrow Z^{1}(\partial, A \rightarrow \\
\left.A^{*}\right) \rightarrow H^{2}(B(T, G, \partial), A) \rightarrow 0,
\end{gathered}
$$

where $D^{*}(\partial, A)$ and $H^{*}(B(T, G, \partial), A)$ are cohomology theories of crossed modules defined in [19] and [13] respectively, and $A \rightarrow A^{*}$ is the canonical injection of $A$ into the coinduced $G$-module $A^{*}$ induced by the $G$-module $A$.

Proof. (i) Let $\varphi_{1}: A_{1} \rightarrow B_{1}, \varphi: A \rightarrow B$ and $\varphi_{2}: A_{2} \rightarrow B_{2}$. Then the commutative 
diagram

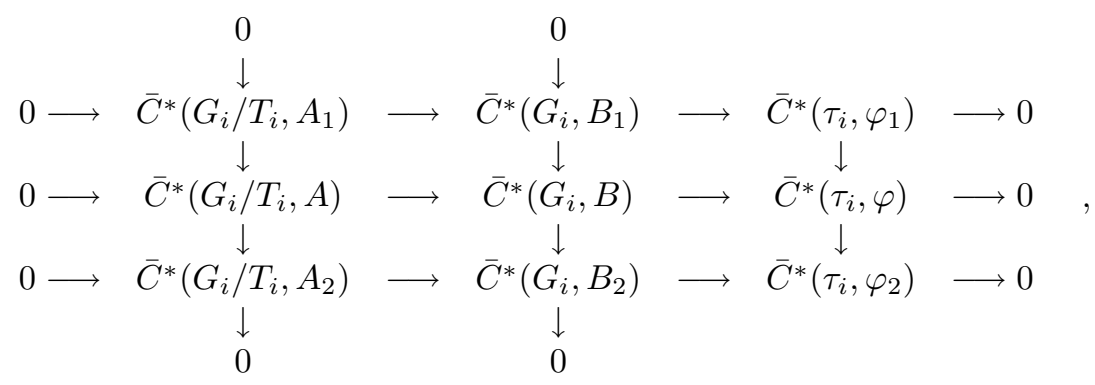

consisting of cochain complexes with exact rows and exact left two columns for $i \geqslant 0$, since the groups $G_{i}$ and $G_{i} / T_{i}$ are free groups, implies the short exact sequence

$$
0 \rightarrow \bar{C}^{*}\left(\tau_{i}, \varphi_{1}\right) \rightarrow \bar{C}^{*}\left(\tau_{i}, \varphi\right) \rightarrow \bar{C}^{*}\left(\tau_{i}, \varphi_{2}\right) \rightarrow 0
$$

for all $i \geqslant 0$.

Hence one gets a short exact sequence of bicomplexes

$$
0 \rightarrow \bar{C}^{*}\left(\tau_{*}, \varphi_{1}\right) \rightarrow \bar{C}^{*}\left(\tau_{*}, \varphi\right) \rightarrow \bar{C}^{*}\left(\tau_{*}, \varphi_{2}\right) \rightarrow 0
$$

implying a short exact sequence of their total cochain complexes

$$
0 \rightarrow \operatorname{Tot} \bar{C}^{*}\left(\tau_{*}, \varphi_{1}\right) \rightarrow \operatorname{Tot} \bar{C}^{*}\left(\tau^{*}, \varphi\right) \rightarrow \operatorname{Tot} \bar{C}^{*}\left(\tau_{*}, \varphi_{2}\right) \rightarrow 0
$$

and therefore giving the required long exact cohomology sequence.

(ii) For any $\tau_{i}: G_{i} \rightarrow G_{i} / T_{i}, i \geqslant 0$, there is a short exact sequence

$$
0 \rightarrow \operatorname{Der}\left(G_{i} / T_{i}, A\right) \rightarrow \operatorname{Der}\left(G_{i}, A\right) \rightarrow \operatorname{Der}\left(\tau_{i}, A\right) \rightarrow 0,
$$

where the groups $G_{i}$ and $G_{i} / T_{i}, i \geqslant 0$, are free groups. Hence we have a short exact sequence of cochain complexes

$$
0 \rightarrow \operatorname{Der}\left(\tau_{*}, A\right) \rightarrow \operatorname{Der}\left(\tau_{*}, \varphi\right) \rightarrow \operatorname{Der}\left(G_{*}, B / \operatorname{Im} \varphi\right) \rightarrow 0
$$

which induces a long exact cohomology sequence

$$
\begin{gathered}
0 \rightarrow \operatorname{Der}(\partial, A) \rightarrow H_{0}\left(\operatorname{Der}\left(\tau_{*}, \varphi\right)\right) \rightarrow \operatorname{Der}(G, B / \operatorname{Im} \varphi) \rightarrow D^{1}(\partial, A) \rightarrow \\
H_{1}\left(\operatorname{Der}\left(\tau_{*}, \varphi\right)\right) \rightarrow H^{2}(G, B / \operatorname{Im} \varphi) \rightarrow D^{2}(\partial, \varphi) \rightarrow \\
H_{2}\left(\operatorname{Der}\left(\tau_{*}, \varphi\right)\right) \rightarrow \cdots \rightarrow H^{n}(G, B / \operatorname{Im} \varphi) \rightarrow \\
D^{n}(\partial, \varphi) \rightarrow H_{n}\left(\operatorname{Der}\left(\tau_{*}, \varphi\right)\right) \rightarrow H^{n+1}(G, B / \operatorname{Im} \varphi) \rightarrow D^{n+1}(\partial, \varphi) \rightarrow \cdots .
\end{gathered}
$$

In the bicomplex $\bar{C}\left(\tau_{*}, \varphi\right)$ the groups $H_{n}\left(\bar{C}^{*}\left(\tau_{i}, \varphi\right)\right)$ are trivial and $H_{0}\left(\bar{C}^{*}\left(\tau_{i}, \varphi\right)\right) \approx \operatorname{Der}\left(\tau_{i}, \varphi\right)$ for all $i \geqslant 0$. It follows that there are isomorphisms $H^{n}(\partial, \varphi) \approx H_{n}\left(\operatorname{Der}\left(\tau_{*}, \varphi\right)\right), n \geqslant 0$, and we get the required long exact cohomology sequence.

Taking as coefficients the injection $\varphi: A \rightarrow A^{*}$ of a $G / \operatorname{Im} \partial$-module $A$ into its coinduced $G$-module $A^{*}=\operatorname{Hom}(\mathbb{Z}[G], A)$ gives rise to a short exact sequence of cochain complexes

$$
0 \rightarrow \operatorname{Der}\left(G_{*} / T_{*}, A\right) \rightarrow \operatorname{Der}\left(G_{*}, A^{*}\right) \rightarrow \operatorname{Der}\left(\tau_{*}, A \rightarrow A^{*}\right) \rightarrow 0
$$


and therefore to a long exact cohomology sequence

$$
\begin{gathered}
0 \rightarrow \operatorname{Der}(G / \operatorname{Im} \partial, A) \rightarrow \operatorname{Der}\left(G, A^{*}\right) \rightarrow Z^{1}\left(\partial, A \rightarrow A^{*}\right) \rightarrow \\
H_{1}\left(\operatorname{Der}\left(G_{*} / T_{*}, A\right)\right) \rightarrow H_{1}\left(\operatorname{Der}\left(G_{*}, A^{*}\right)\right) \rightarrow \cdots H_{n}\left(\operatorname{Der}\left(G_{*}, A^{*}\right)\right) \rightarrow \\
H_{n}\left(\operatorname{Der}\left(\tau_{*}, A \rightarrow A^{*}\right)\right) \rightarrow H_{n+1}\left(\operatorname{Der}\left(G_{*} / T_{*}\right)\right) \rightarrow H_{n+1}\left(\operatorname{Der}\left(G_{*}, A^{*}\right)\right) \rightarrow \cdots .
\end{gathered}
$$

As we have seen $H^{n}\left(\partial, A \rightarrow A^{*}\right)$ is isomorphic to $H_{n}\left(\operatorname{Der}\left(\tau_{*}, A \rightarrow A^{*}\right)\right), n \geqslant 0$. On the other hand, the groups $H_{n}\left(\operatorname{Der}\left(G_{*}, A^{*}\right)\right)$ are isomorphic to $H^{n+1}\left(G, A^{*}\right)$ for $n \geqslant 1$, since $G_{*} \rightarrow G$ is a projective resolution of the group $G$, and therefore are trivial for $n \geqslant 1$. Finally, in [19] it is proved that the groups $H_{n}\left(\operatorname{Der}\left(G_{*} / T_{*}, A\right)\right)$ are isomorphic to the cohomology groups $H^{n+1}(B(T, G, \partial), A), n \geqslant 1$, of crossed modules defined in [13]. Now from the above long exact cohomology sequence we can deduce the last two statements of (ii).

Note that in [19] it is proved that the cohomology theory $D^{*}(\partial, A)$ of crossed modules is closely related with the cohomology theories of crossed modules defined in [5] and [13].

Define the group $Z^{k}(\partial, \varphi)$ as $H_{0}\left(Z^{k}\left(\tau_{*}, \varphi\right)\right)$ for any crossed module $\partial$ acting on $\varphi$ and all $k \geqslant 1$. Clearly for $k=1$ we recover the above definition of $Z^{1}(\partial, \varphi)$. It is also easily seen that if $\tau: G \rightarrow G^{\prime}$ is a surjective group homomorphism acting on $\varphi$, the group $Z^{k}(\operatorname{Ker} \tau \hookrightarrow G, \varphi)$ is isomorphic to $Z^{k}(\tau, \varphi), k \geqslant 1$.

Theorem 19. There is a natural isomorphism

$$
R_{\mathcal{G}}^{n} Z^{1}(\partial, \varphi) \approx H^{n}(\partial, \varphi) \text { for all } n>0
$$

and a long exact sequence

$$
\begin{gathered}
0 \rightarrow Z^{k}(\partial, \varphi) \rightarrow H_{0}\left(C^{k}\left(\tau_{*}, \varphi\right)\right) \rightarrow Z^{k+1}(\partial, \varphi) \rightarrow R_{\mathcal{G}}^{1} Z^{k}(\partial, \varphi) \rightarrow \\
H_{2}\left(C^{k}\left(G_{*} / T_{*}, A\right)\right) \rightarrow R_{\mathcal{G}}^{1} Z^{k+1}(\partial, \varphi) \rightarrow \cdots \rightarrow R_{\mathcal{G}}^{n} Z^{k+1}(\partial, \varphi) \rightarrow R_{\mathcal{G}}^{n+1} Z^{k}(\partial, \varphi) \rightarrow \\
H_{n+2}\left(C^{k}\left(G_{*} / T_{*}, A\right)\right) \rightarrow R_{\mathcal{G}}^{n+1} Z^{k+1}(\partial, \varphi) \rightarrow R_{\mathcal{G}}^{n+1} Z^{k}(\partial, \varphi) \rightarrow \cdots
\end{gathered}
$$

for all $k \geqslant 1$.

Proof. Since the groups $G_{i}$ and $G_{i} / T_{i}$ are free groups, $i \geqslant 0$, from the short exact sequence of cochain complexes

$$
0 \rightarrow \bar{C}^{*}\left(G_{i} / T_{i}, A\right) \rightarrow \bar{C}^{*}\left(G_{i}, B\right) \rightarrow \bar{C}^{*}\left(\tau_{i}, \varphi\right) \rightarrow 0, i \geqslant 0
$$

it follows that $H_{n}\left(\bar{C}^{*}\left(\tau_{i}, \varphi\right)\right)=0$ for $n>0$, and $H_{0}\left(\bar{C}^{*}\left(\tau_{i}, \varphi\right)\right)=Z^{1}\left(\tau_{i}, \varphi\right), i \geqslant 0$. Therefore the homology group $H_{n}\left(\operatorname{Tot} \bar{C}^{*}\left(\tau_{*}, \varphi\right)\right)$ is isomorphic to $H_{n}\left(Z^{1}\left(\tau_{*}, \varphi\right)\right)$ for $n \geqslant 0$.

The short exact sequence of cochain complexes

$$
0 \rightarrow C^{k}\left(G_{*} / T_{*}, A\right) \rightarrow C^{k}\left(G_{*}, B\right) \rightarrow C^{k}\left(\tau_{*}, \varphi\right) \rightarrow 0
$$

induces an isomorphism $H_{n}\left(C^{k}\left(\tau_{*}, \varphi\right)\right) \approx H_{n+1}\left(C^{k}\left(G_{*} / T_{*}, A\right)\right)$ for $n, k \geqslant 1$, because $G_{*} \rightarrow G$ as a projective resolution of $G$ is simplicially exact and therefore left (right) contractible as augmented simplicial set, implying the triviality of the groups $H_{n}\left(C^{k}\left(G_{*}, B\right)\right)$ for $n>0$. On the other hand, for $k \geqslant 1$ one has the following 
short exact sequence of cochain complexes:

$$
0 \rightarrow Z^{k}\left(\tau_{*}, \varphi\right) \rightarrow C^{k}\left(\tau_{*}, \varphi\right) \rightarrow Z^{k+1}\left(\tau_{*}, \varphi\right) \rightarrow 0,
$$

inducing a long exact sequence

$$
\begin{gathered}
0 \rightarrow Z^{k}(\partial, \varphi) \rightarrow H_{0}\left(C^{k}\left(\tau_{*}, \varphi\right)\right) \rightarrow Z^{k+1}(\partial, \varphi) \rightarrow R_{\mathcal{G}}^{1} Z^{k}(\partial, \varphi) \rightarrow H_{1}\left(C^{k}\left(\tau_{*}, \varphi\right)\right) \rightarrow \\
R_{\mathcal{G}}^{1} Z^{k+1}(\partial, \varphi) \rightarrow \cdots R_{\mathcal{G}}^{n} Z^{k+1}(\partial, \varphi) \rightarrow R_{\mathcal{G}}^{n+1} Z^{k}(\partial, \varphi) \rightarrow \\
H_{n+1}\left(C^{k}\left(\tau_{*}, \varphi\right)\right) \rightarrow R_{\mathcal{G}}^{n+1} Z^{k+1}(\partial, \varphi) \rightarrow R_{\mathcal{G}}^{n+1} Z^{k}(\partial, \varphi) \cdots
\end{gathered}
$$

This completes the proof.

\section{References}

[1] M.Barr and J.Beck, Acyclic models and triples, Proc. of La Jolla Conference on Categorical Algebra, Springer-Verlag, New York (1966), 336-343.

[2] M.Barr and J.Beck, Homology and standard constructions, Lecture Notes in Math., Springer-Verlag, vol.80 (1969), 245-335.

[3] M.Barr and G.Reinhart, Cohomology as the derived functor of derivations, Trans.Amer.Math.Soc. 122 (1966), 416-425.

[4] J.Beck, Triples, algebras and cohomology, Dissertation, Columbia University, 1964-67.

[5] P.Carrasco, A.M.Cegarra and A.R.Grandjean, (Co)homology of crossed modules, Elsevier Preprint, 1999.

[6] R.Brown and G.Ellis, Hopf formulae for the higher homology of a group, Bull.London Math.Soc. 20 (1988), 124-128.

[7] A.M.Cegarra, J.M.Garcia-Calcines and J.A.Ortega, Cohomology of groups with operators, Homology, Homotopy and Applications 4 (1) (2002), 1-23.

[8] A.M.Cegarra, J.M.Garcia-Calcines and J.A.Ortega, On graded categorical groups and equivariant group extensions, Canad. J. Math. 54 (5) (2002), 970-997.

[9] D.Conduche, H.Inassaridze and N.Inassaridze, Mod q cohomology and Tate cohomology of groups, Prepublication 01-29, Institut de Recherche Mathematique de Rennes, 2001.

[10] D.Conduche, H.Inassaridze and N.Inassaridze, Mod $q$ cohomology and Tate-Vogel cohomology of groups, J.Pure Appl.Algebra, 189 (2004), 6187.

[11] B.Conrad, Crossed $n$-fold extensions of groups, $n$-fold extensions of modules and higher multipliers, J.Pure Appl.Algebra 36 (1985), 225-235.

[12] G.Donadze, N.Inassaridze and T.Porter, $N$-fold Čech derived functors and generalised Hopf type formulas, K-theory Preprint Archive 2003 (http://www.math.uiuc.edu/K-theory/0624/). 
[13] G.J.Ellis, Homology of 2-types, J.London Math.Soc. 46, (2) (1992), 1-27.

[14] A.Frohlich and C.T.C.Wall, Graded monoidal categories, Comp.Math. 28 (1974), 229-285.

[15] H.Inassaridze, Non-abelian homological algebra and its applications, Kluwer Acad. Publishers, Amsterdam, 1997.

[16] H.Inassaridze, Equivariant homology and cohomology of groups, Topology and its Applications,2004 (to appear)(Math.Prep. Server, 2003, http://www.mathpreprints.com).

[17] J.-L.Loday, Cohomologie et groupe de Steinberg relatifs, J.Algebra 54 (1978), 178-202.

[18] J.-L.Loday, Cyclic homology, Springer, 1998.

[19] S.Paoli, (Co)homology of crossed modules with coefficients in a $\pi_{1}$ - module, Homology, Homotopy and Applications, 5 (1) (2003), 261-296.

[20] A.Rodicio, Presentaciones libres y $H_{2 n}(G)$, Publ.Mat.Univ.Aut.Barcelona 30 (1986), 77-82.

[21] R.Stohr, A generalized Hopf formula for higher homology groups, Comment.Math.Helvetici 64 (1989), 187-199.

[22] R.Swan, Some relations between higher K-functors, J.Algebra 21, (1) (1972), 113-136.

[23] M.Tierney and W.Vogel, Simplicial resolutions and derived functors, Math.Zeit. 111 (1969), 1-14.

This article may be accessed via WWW at http://www.rmi.acnet.ge/hha/ or by anonymous ftp at

ftp://ftp.rmi.acnet.ge/pub/hha/volumes/2005/n1a6/v7n1a6.(dvi,ps,pdf)

Hvedri Inassaridze hvedri@rmi.acnet.ge

A.Razmadze Mathematical Institute, Georgian Academy of Sciences,

M. Alexidze Str. 1,

Tbilisi 0193,

Georgia 